\title{
Gevrey Asymptotic Theory for Singular First Order Linear Partial Differential Equations of Nilpotent Type \\ - Part II -
}

By

Masaki HiBıno*

\section{$\S 1 . \quad$ Introduction and Main Results}

In this paper, as a continuation of the previous paper [6] which is referred as "Part I" throughout this paper, we are concerned with the Borel summability of formal solutions for the following first order linear partial differential equation of nilpotent type:

$$
\begin{gathered}
L u(x, y)=f(x, y), \\
L=1+\left(\mathrm{a} y+\mathrm{b} x y+\mathrm{c} y^{2}\right) D_{x}+\mathrm{d} y^{2} D_{y},
\end{gathered}
$$

where $x, y \in \mathbf{C}, D_{x}=\partial / \partial x, D_{y}=\partial / \partial y$, and $\mathrm{a}, \mathrm{b}, \mathrm{c}$ and $\mathrm{d}$ are complex constants, and $f(x, y)$ is holomorphic at the origin. Throughout this paper, we always assume that

$$
a \neq 0 \text {. }
$$

The reason why we consider this type of equation will be explained in the end of this section.

As mentioned in Part I, we know that the equation (1.1) has a unique formal power series solution in $\mathcal{O}[R][[y]]_{2}$ for some $R>0$. Here we say that a formal power series $u(x, y)$ belongs to $\mathcal{O}[R][[y]]_{2}$ if $u(x, y)$ can be written as $u(x, y)=\sum_{n=0}^{\infty} u_{n}(x) y^{n}$, where all $u_{n}(x)$ are holomorphic on $\{x \in \mathbf{C} ;|x| \leq R\}$ with the estimates $\max _{|x| \leq R}\left|u_{n}(x)\right| \leq C K^{n} n$ !. Therefore the formal solution of (1.1) is divergent in general.

Communicated by T. Kawai, December 5, 2000. Revised April 2, 2001.

2000 Mathematics Subject Classification(s): Primary 35C20, Secondary 35C10, 35C15.

*Graduate School of Mathematics, Nagoya University, Nagoya 464-8602, Japan.

e-mail: m96006y@math.nagoya-u.ac.jp 
The purpose of this paper (and Part I as well) is to prove the existence of a holomorphic solution which has this divergent solution $u(x, y) \in \mathcal{O}[R][[y]]_{2}$ as asymptotic expansion. We have two types of asymptotic expansions: "asymptotic expansion in a small sector" and "Borel summability". Since the asymptotic expansion in a small sector was studied in Part I (see the subsection 1.3 also), we will study the Borel summability in this paper as stated above.

Now let us define the concept of our asymptotic expansion which is called the Borel summability.

Definition 1.1. (1) For $\theta \in \mathbf{R}$ and $Y>0$, we define the region $O(\theta, Y)$ by

$$
O(\theta, Y)=\left\{y \in \mathbf{C} ;\left|y-Y e^{i \theta}\right|<Y\right\} .
$$

(2) Let $u(x, y)=\sum_{n=0}^{\infty} u_{n}(x) y^{n} \in \mathcal{O}[R][[y]]_{2}$. We say that $u(x, y)$ is Borel summable in $\theta$-direction if there exists a holomorphic function $w(x, y)$ on $\{x \in \mathbf{C} ;|x| \leq r\} \times O(\theta, Y)$ for some $r>0$ and $Y>0$ which satisfies the following asymptotic estimates: There exist some positive constants $C$ and $K$ such that

$$
\max _{|x| \leq r}\left|w(x, y)-\sum_{n=0}^{N-1} u_{n}(x) y^{n}\right| \leq C K^{N} N !|y|^{N},
$$

for $y \in O(\theta, Y)$ and $N=1,2, \ldots$

When $u(x, y)$ is Borel summable in $\theta$-direction, the above function $w(x, y)$ is unique (see Lutz-Miyake-Schäfke [7]). Therefore we call this $w(x, y)$ the Borel sum of $u(x, y)$ in $\theta$-direction.

In this paper we study the condition under which the formal solution of (1.1) is Borel summable. The case $\mathrm{c}=0$ has been already studied in Part I. Before stating our main result, let us recall some results obtained in Part I.

\section{§1.1. Results in Part I}

In this subsection, we assume $\mathrm{c}=0$. First, we divide the problem into the following four cases:

Case (1): $b=d=0$,

Case (2): $\mathrm{b}=0, \mathrm{~d} \neq 0$,

Case (3): $b \neq 0, d=0$,

Case (4): $b, d \neq 0$.

Now in order to state results in Part I in a specific manner, let us introduce 
some notations. We define the function $\Phi(x, \eta)$ by

$$
\Phi(x, \eta)= \begin{cases}x-\mathrm{a} \eta & (\text { Case (1)) } \\ x-\frac{\mathrm{a}}{\mathrm{d}} \log (1+\mathrm{d} \eta) & (\text { Case }(2)) \\ \left(\frac{\mathrm{a}}{\mathrm{b}}+x\right) e^{-\mathrm{b} \eta}-\frac{\mathrm{a}}{\mathrm{b}} & (\text { Case }(3)) \\ \left(\frac{\mathrm{a}}{\mathrm{b}}+x\right)(1+\mathrm{d} \eta)^{-\mathrm{b} / \mathrm{d}}-\frac{\mathrm{a}}{\mathrm{b}} & (\text { Case }(4)),\end{cases}
$$

and define the region $\Omega_{r, \theta, \rho} \subset \mathbf{C}$ by

$$
\Omega_{r, \theta, \rho}=\Phi\left(\left\{(x, \eta) \in \mathbf{C} ;|x| \leq r, \eta \in E_{+}(\theta, \rho)\right\}\right) .
$$

Here $E_{+}(\theta, \rho)$ is a region defined by

$$
E_{+}(\theta, \rho)=\left\{\eta \in \mathbf{C} ; \operatorname{dist}\left(\eta, \mathbf{R}_{+} e^{i \theta}\right) \leq \rho\right\},
$$

where $\mathbf{R}_{+}=[0,+\infty)$.

In Case (2) and Case (4), we assume $\theta \neq \arg (-1 / \mathrm{d})$ so that $\Omega_{r, \theta, \rho}$ is welldefined. In Case (3) and Case (4), we remark that $\Omega_{r, \theta, \rho}$ is a region in the Riemann surface of $\log \left(x+\frac{\mathrm{a}}{\mathrm{b}}\right)$.

We remark that $\Phi(x, \eta)$ in Cases (1), (2) and (3) are obtained by taking a limit from $\Phi(x, \eta)$ in Case (4) as shown in the following diagram:

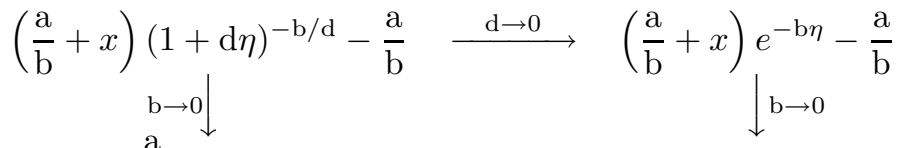

$$
\begin{aligned}
& x-\frac{\mathrm{a}}{\mathrm{d}} \log (1+\mathrm{d} \eta) \quad \underset{\mathrm{d} \rightarrow 0}{\longrightarrow} \quad x-\mathrm{a} \eta .
\end{aligned}
$$

In Part I, we obtained the following theorem:

Theorem 1.1 (Hibino [6]). Let $\theta \in \mathbf{R}$ and suppose that $\theta \neq \arg (-1 / \mathrm{d}$ ) in Case (2) and Case (4). Assume that $f(x, y)$ can be continued analytically to $\left\{(x, y) \in \mathbf{C}^{2} ; x \in \Omega_{r, \theta, \rho},|y| \leq r^{\prime}\right\}$ for some $r, \rho$ and $r^{\prime}$ in each case. We further assume that $f(x, y)$ has the following growth estimate with some positive constants $C$ and $\delta:$ For $x \in \Omega_{r, \theta, \rho}$,

Case (1):

$$
\max _{|y| \leq r^{\prime}}|f(x, y)| \leq C e^{\delta|x|} ;
$$

Case (2):

$$
\max _{|y| \leq r^{\prime}}|f(x, y)| \leq C \exp \left(\delta e^{p|x|}\right),
$$


where $p=|\mathrm{d} / \mathrm{a}|$;

Case (3):

$$
\max _{|y| \leq r^{\prime}}|f(x, y)| \leq C \exp \left\{\delta\left|\log \left(x+\frac{\mathrm{a}}{\mathrm{b}}\right)\right|\right\}
$$

Case (4):

$$
\max _{|y| \leq r^{\prime}}|f(x, y)| \leq C \exp \left[\delta \exp \left\{\left|\frac{\mathrm{d}}{\mathrm{b}}\right|\left|\log \left(x+\frac{\mathrm{a}}{\mathrm{b}}\right)\right|\right\}\right] .
$$

Then the formal solution $u(x, y)$ of the equation (1.1) with $\mathrm{c}=0$ is Borel summable in $\theta$-direction and its Borel sum is a holomorphic solution of (1.1).

\section{$\S 1.2$. Main result}

In this paper, we want to remove the condition $\mathrm{c}=0$. To achieve this, we need some additional conditions for Cases (3) and (4). Let us use the same notations as in the previous subsection. Then our main result is stated as follows:

Theorem 1.2. Assume that $f(x, y)$ satisfies the same condition as in Theorem 1.1. Furthermore in Cases (3) and (4), we assume the following condition:

Case (3):

$$
\mathrm{c}=0 \quad \text { or } \quad \Re\left(-\mathrm{b} e^{i \theta}\right) \geq 0 ;
$$

Case (4):

$$
\mathrm{c}=0 \quad \text { or } \Re\left(-\frac{\mathrm{b}}{\mathrm{d}}\right)>-1 .
$$

Then we have the same conclusion as in Theorem 1.1 for each case.

We will prove this theorem in the Sections 3 through 6 . In the proof, we consider an integro-differential equation (the equation (2.5) below) which is obtained by applying the formal Borel transformation to (1.1), and prove an analytic continuation property and an exponential growth estimate for solutions of (2.5) by using the iteration method. For each case, we have to prove the different type of iteration estimates (cf. the estimates (3.9), (4.9), (5.9) and $(6.10))$. We remark that the function $\Phi(x, \eta)$ exactly describes the characteristic curve of the first order linear partial differential operator which appears in the left hand side of (2.5). 


\section{$\S 1.3 . \quad$ Some remarks}

Here we give some remarks for the precedent results.

(1) When $\mathrm{c}=0$ and $f(x, y)$ is a polynomial with respect to $y$, we can obtain the necessary and sufficient condition for the Borel summability (see Part I). In this case, it is not necessary to require the analytic continuation property for $f(x, y)$ itself (cf. Theorem 3.2 in [6]).

(2) In the proof of Theorem 1.1 and 1.2, we use Theorem 2.1 (see LutzMiyake-Schäfke [7]) as a fundamental tool. The paper [7] is the first one which considered the Borel summability for solutions of partial differential equations. For ordinary differential equations there are many results which can be seen in Balser [1], [2] and [7] gave the necessary and sufficient condition for the Borel summability of the formal solution of the heat equation.

(3) In Part I, we also studied the existence of asymptotic solutions in a small sector. Even if the coefficients a, b, c and d are holomorphic functions, we can prove the existence of an asymptotic solution by using the Gevrey version of Borel-Ritt's Theorem (see Balser [2]). We remark that neither the original version nor the Gevrey version of Borel-Ritt's Theorem is useful in discussing the Borel summability.

Finally we briefly explain the reason why we consider this nilpotent type of equation. The word "nilpotent" means that the Jacobi matrix at the origin of the coefficients of the principal part of (1.1) is a nilpotent matrix. Singular first order equations have been studied, for example, in Gérard-Tahara [4] and Oshima [12], but they discuss the case where formal solutions converge, assuming that the Jordan canonical form of the Jacobi matrix has no nilpotent Jordan block (though in their studies, the Jacobi matrix has non-zero eigenvalues instead). The divergence of formal solutions for our equations is caused by the nilpotent part and therefore, as one of the generalizations of these works, it is natural to investigate the asymptotic theory for these divergent solutions.

\section{$\S 2$. Formal Borel Transform of Equations}

Before proving Theorem 1.2, we give some preliminaries. First, we remark that if the formal solution $u(x, y)$ of (1.1) is Borel summable, then its Borel sum $w(x, y)$ is a holomorphic solution of (1.1). It is easily proved by using the uniqueness of the Borel sum (for the detail, see Part I).

Thus in order to prove Theorem 1.2, it is sufficient to prove that the formal solution $u(x, y)$ is Borel summable under the conditions in the theorem.

When we want to check the Borel summability of a formal power series 
$u(x, y)=\sum_{n=0}^{\infty} u_{n}(x) y^{n} \in \mathcal{O}[R][[y]]_{2}$, the following theorem plays a fundamental role in general.

Theorem 2.1 (Lutz, Miyake and Schäfke [7]). In order that a formal power series $u(x, y)=\sum_{n=0}^{\infty} u_{n}(x) y^{n} \in \mathcal{O}[R][[y]]_{2}$ is Borel summable in $\theta$-direction, the following condition (BS) is necessary and sufficient: Let $v(x, \eta)$ be the formal Borel transform of $u(x, y)$ defined by

$$
v(x, \eta)=\sum_{n=0}^{\infty} u_{n}(x) \frac{\eta^{n}}{n !},
$$

which is holomorphic in a neighborhood of the origin. Then the condition (BS) is stated as follows:

(BS) $v(x, \eta)$ can be continued analytically to $\{x \in \mathbf{C} ;|x| \leq r\} \times E_{+}(\theta, \rho)$ for some $r>0$ and $\rho>0$, and has the following exponential growth estimate for some positive constants $C$ and $\delta$ :

$$
\max _{|x| \leq r}|v(x, \eta)| \leq C e^{\delta|\eta|}, \quad \eta \in E_{+}(\theta, \rho) .
$$

When (BS) is satisfied, the Borel sum $w(x, y)$ of $u(x, y)$ in $\theta$-direction is given by

$$
w(x, y)=\frac{1}{y} \int_{\mathbf{R}_{+} e^{i \theta}} e^{-\eta / y} v(x, \eta) d \eta
$$

It is thus sufficient to prove that the formal Borel transform $v(x, \eta)$ of the formal solution $u(x, y)$ satisfies the above condition (BS) under the conditions in the theorem. In order to do that, firstly let us write down the equation which $v(x, \eta)$ should satisfy. By the formal Borel transform, the operators $y$ and $D_{y}$ are transformed to the operators $D_{\eta}^{-1}=\int_{0}^{\eta} d \eta$ and $D_{\eta} \eta D_{\eta}$, respectively (cf. the commutative diagrams in the Section 3.1 of Part I. Similar type of diagrams can be seen also in [7]).

Therefore we see that $v(x, \eta)$ is a solution of the following equation:

$$
\left\{1+(\mathrm{a}+\mathrm{b} x) D_{\eta}^{-1} D_{x}+\mathrm{c} D_{\eta}^{-2} D_{x}+\mathrm{d} D_{\eta}^{-1} \eta D_{\eta}\right\} v(x, \eta)=g(x, \eta)
$$

where $g(x, \eta)$ is the formal Borel transform of $f(x, y)=\sum_{n=0}^{\infty} f_{n}(x) y^{n}$, that is,

$$
g(x, \eta)=\sum_{n=0}^{\infty} f_{n}(x) \frac{\eta^{n}}{n !} .
$$


Furthermore by operating $D_{\eta}$ to the equation (2.4) from the left, we see that $v(x, \eta)$ is a solution of the initial value problem of the following integro-differential equation:

$$
\begin{gathered}
\left\{(1+\mathrm{d} \eta) D_{\eta}+(\mathrm{a}+\mathrm{b} x) D_{x}\right\} v(x, \eta)=-\mathrm{c} D_{\eta}^{-1} D_{x} v(x, \eta)+h(x, \eta) \\
v(x, 0)=f(x, 0)
\end{gathered}
$$

where $h(x, \eta)=D_{\eta} g(x, \eta)$.

Theorem 1.2 will be proved by showing that the solution $v(x, \eta)$ of the equation (2.5) satisfies the condition (BS).

\section{§3. The Proof of Theorem 1.2 (Case (1))}

In Case (1) $(b=d=0)$, the equation (2.5) is written as follows:

$$
\begin{gathered}
\left\{D_{\eta}+\mathrm{a} D_{x}\right\} v(x, \eta)=-\mathrm{c} D_{\eta}^{-1} D_{x} v(x, \eta)+h(x, \eta), \\
v(x, 0)=f(x, 0) .
\end{gathered}
$$

We will prove that the solution $v(x, \eta)$ of (3.1) satisfies the condition (BS) in Theorem 2.1. First, we remark that in general the solution $w(x, \eta)$ of the initial value problem of the following first order linear partial differential equation

$$
\begin{gathered}
\left\{D_{\eta}+\mathrm{a} D_{x}\right\} w(x, \eta)=k(x, \eta), \\
w(x, 0)=l(x)
\end{gathered}
$$

is given by

$$
w(x, \eta)=l(x-\mathrm{a} \eta)+\int_{0}^{\eta} k(x-\mathrm{a}(\eta-t), t) d t .
$$

Proof of the theorem. Since the theorem has been already proved in the case $c=0$, we assume that $c \neq 0$. In this case, the equation (3.1) is rewritten as follows:

$$
\begin{gathered}
\left\{D_{\eta}+\mathrm{a} D_{x}\right\} v(x, \eta)=-\mathrm{c} \int_{0}^{\eta} v_{x}(x, s) d s+h(x, \eta), \\
v(x, 0)=f(x, 0) .
\end{gathered}
$$

First, let us transform (3.4) into the integral equation. It follows from (3.3) that the equation (3.4) is equivalent to the following equation:

$v(x, \eta)=f(x-\mathrm{a} \eta, 0)+\int_{0}^{\eta} h(x-\mathrm{a}(\eta-t), t) d t-\mathrm{c} \int_{0}^{\eta} \int_{0}^{t} v_{x}(x-\mathrm{a}(\eta-t), s) d s d t$. 
Here we remark that

$$
\begin{aligned}
& \int_{0}^{\eta} \int_{0}^{t} v_{x}(x-\mathrm{a}(\eta-t), s) d s d t \\
& =\int_{0}^{\eta} \int_{s}^{\eta} v_{x}(x-\mathrm{a}(\eta-t), s) d t d s \\
& =\int_{0}^{\eta} \int_{s}^{\eta} \frac{d}{d t}\left\{\frac{1}{\mathrm{a}} v(x-\mathrm{a}(\eta-t), s)\right\} d t d s \\
& =\frac{1}{\mathrm{a}} \int_{0}^{\eta} v(x, t) d t-\frac{1}{\mathrm{a}} \int_{0}^{\eta} v(x-\mathrm{a}(\eta-t), t) d t .
\end{aligned}
$$

Therefore we know that (3.4) is equivalent to the following integral equation:

$$
\begin{aligned}
v(x, \eta)= & f(x-\mathrm{a} \eta, 0)+\int_{0}^{\eta} h(x-\mathrm{a}(\eta-t), t) d t \\
& +\frac{\mathrm{c}}{\mathrm{a}} \int_{0}^{\eta} v(x-\mathrm{a}(\eta-t), t) d t-\frac{\mathrm{c}}{\mathrm{a}} \int_{0}^{\eta} v(x, t) d t .
\end{aligned}
$$

In order to prove that the solution $v(x, \eta)$ of $(3.5)$ satisfies the condition (BS), we employ the iteration method. Let us define $\left\{v_{n}(x, \eta)\right\}_{n=0}^{\infty}$ as follows:

$$
v_{0}(x, \eta)=f(x-\mathrm{a} \eta, 0)+\int_{0}^{\eta} h(x-\mathrm{a}(\eta-t), t) d t .
$$

For $n \geq 0$,

$$
v_{n+1}(x, \eta)=v_{0}(x, \eta)+\frac{\mathrm{c}}{\mathrm{a}} \int_{0}^{\eta} v_{n}(x-\mathrm{a}(\eta-t), t) d t-\frac{\mathrm{c}}{\mathrm{a}} \int_{0}^{\eta} v_{n}(x, t) d t .
$$

Next we put $w_{0}(x, \eta):=v_{0}(x, \eta)$ and $w_{n}(x, \eta):=v_{n}(x, \eta)-v_{n-1}(x, \eta)$ for $n \geq 1$, and we define $\widetilde{w}_{n}(x, \eta, t)$ by

$$
\widetilde{w}_{n}(x, \eta, t):=w_{n}(x-\mathrm{a}(\eta-t), t) .
$$

Definition 3.1. (i) For $\alpha \geq 0$ and $\varepsilon>0, U_{\varepsilon}[0, \alpha]$ denotes the $\varepsilon-$ neighborhood of $[0, \alpha]$ in $\mathbf{C}$.

(ii) For $\eta \in \mathbf{C}$, we define a function $G_{\eta}(\tau)$ by

$$
G_{\eta}(\tau)=\tau e^{i \arg (\eta)}, \quad \tau \in \mathbf{C}
$$

and we put

$$
\begin{aligned}
G_{\eta}:= & \left\{G_{\eta}(R) \in \mathbf{C} ; 0 \leq R \leq|\eta|\right\}, \\
& G_{\eta}^{\varepsilon}:=G_{\eta}\left(U_{\varepsilon}[0,|\eta|]\right) .
\end{aligned}
$$

We remark that $G_{\eta}$ is the segment from 0 to $\eta$ and that $G_{\eta}^{\varepsilon}$ is the $\varepsilon$-neighbor- 
$\operatorname{hood}$ of $G_{\eta}$.

Now let us take a monotonically decreasing positive sequence $\left\{\varepsilon_{n}\right\}_{n=0}^{\infty}$ satisfying

$$
\widetilde{\rho}:=\rho-\sum_{n=0}^{\infty} \varepsilon_{n}>0 .
$$

Then we have the following lemma:

Lemma 3.1. $\quad \widetilde{w}_{n}(x, \eta, t)$ is continued analytically to $\left\{(x, \eta, t) \in \mathbf{C}^{3} ;|x|\right.$ $\left.\leq r, \eta \in E_{+}\left(\theta, \rho-\sum_{j=0}^{n} \varepsilon_{j}\right), t \in G_{\eta}^{\varepsilon_{n}}\right\}$. Furthermore on $\left\{(x, \eta, t) \in \mathbf{C}^{3} ;|x| \leq\right.$ $\left.r, \eta \in E_{+}\left(\theta, \rho-\sum_{j=0}^{n} \varepsilon_{j}\right), t \in G_{\eta}\right\}$ we have the following estimate: For some positive contants $C_{1}$ and $\delta_{1}$,

$$
\widetilde{w}_{n}\left(x, \eta, G_{\eta}(R)\right) \mid \leq C_{1} e^{\delta_{1}|\eta|} L^{n} \sum_{k=0}^{n}\left(\begin{array}{l}
n \\
k
\end{array}\right) \frac{1}{\delta_{1}^{n-k}} \frac{R^{k}}{k !},
$$

where $L=|\mathrm{c}| /|\mathrm{a}|$.

If we admit Lemma 3.1, the theorem is proved as follows: It follows from Lemma 3.1 that $w_{n}(x, \eta)\left(=\widetilde{w}_{n}(x, \eta, \eta)\right)$ is continued analytically to $\{(x, \eta) \in$ $\left.\mathbf{C}^{2} ;|x| \leq r, \eta \in E_{+}\left(\theta, \rho-\sum_{j=0}^{n} \varepsilon_{j}\right)\right\}$ with the estimate

$$
\begin{aligned}
\left|w_{n}(x, \eta)\right| & =\left|\widetilde{w}_{n}\left(x, \eta, G_{\eta}(|\eta|)\right)\right| \\
& \leq C_{1} e^{\delta_{1}|\eta|} L^{n} \sum_{k=0}^{n}\left(\begin{array}{l}
n \\
k
\end{array}\right) \frac{1}{\delta_{1}^{n-k}} \frac{|\eta|^{k}}{k !},
\end{aligned}
$$

for $|x| \leq r$ and $\eta \in E_{+}\left(\theta, \rho-\sum_{j=0}^{n} \varepsilon_{j}\right)$. Therefore $v_{n}(x, \eta)\left(=\sum_{k=0}^{n} w_{k}(x, \eta)\right)$ converges to a solution $V(x, \eta)$ of (3.5) uniformly on $\left\{(x, \eta) \in \mathbf{C}^{2} ;|x| \leq r, \eta \in\right.$ $\left.E_{+}(\theta, \widetilde{\rho})\right\}$ as will be shown below. On the other hand, since $v(x, \eta)$ is a local holomorphic solution of (3.5), it follows from the uniqueness of local holomorphic solutions that $V(x, \eta)$ is the analytic continuation of $v(x, \eta)$. Furthermore on $\left\{(x, \eta) \in \mathbf{C}^{2} ;|x| \leq r, \eta \in E_{+}(\theta, \widetilde{\rho})\right\}$ it holds that

$$
\begin{aligned}
|V(x, \eta)| & \leq \sum_{n=0}^{\infty}\left|w_{n}(x, \eta)\right| \\
& \leq C_{1} e^{\delta_{1}|\eta|} \sum_{n=0}^{\infty} L^{n} \sum_{k=0}^{n}\left(\begin{array}{l}
n \\
k
\end{array}\right) \frac{1}{\delta_{1}^{n-k}} \frac{|\eta|^{k}}{k !} \\
& =C_{1} e^{\delta_{1}|\eta|} \sum_{k=0}^{\infty} \sum_{n=k}^{\infty} L^{n}\left(\begin{array}{l}
n \\
k
\end{array}\right) \frac{1}{\delta_{1}^{n}} \frac{\left(\delta_{1}|\eta|\right)^{k}}{k !} .
\end{aligned}
$$


Here we may take $\delta_{1}>0$ so large that

$$
\delta_{1}>2 L
$$

Hence we have

$$
\sum_{n=k}^{\infty} L^{n}\left(\begin{array}{l}
n \\
k
\end{array}\right) \frac{1}{\delta_{1}^{n}} \leq \sum_{n=k}^{\infty}\left(\frac{2 L}{\delta_{1}}\right)^{n}=\left(\frac{2 L}{\delta_{1}}\right)^{k} \frac{1}{1-2 L / \delta_{1}}=\left(\frac{2 L}{\delta_{1}}\right)^{k} \frac{\delta_{1}}{\delta_{1}-2 L} .
$$

Therefore it holds that

$$
\begin{aligned}
|V(x, \eta)| & \leq \frac{C_{1} \delta_{1}}{\delta_{1}-2 L} e^{\delta_{1}|\eta|} \sum_{k=0}^{\infty}\left(\frac{2 L}{\delta_{1}}\right)^{k} \frac{\left(\delta_{1}|\eta|\right)^{k}}{k !} \\
& =\frac{C_{1} \delta_{1}}{\delta_{1}-2 L} e^{\delta_{1}|\eta|} \sum_{k=0}^{\infty} \frac{(2 L|\eta|)^{k}}{k !} \\
& =\frac{C_{1} \delta_{1}}{\delta_{1}-2 L} e^{\delta_{1}|\eta|} e^{2 L|\eta|}
\end{aligned}
$$

which implies

$$
\max _{|x| \leq r}|V(x, \eta)| \leq \widetilde{C} e^{\widetilde{\delta}|\eta|}
$$

for $\eta \in E_{+}(\theta, \widetilde{\rho})$, where $\widetilde{C}=C_{1} \delta_{1} /\left(\delta_{1}-2 L\right)$ and $\widetilde{\delta}=\delta_{1}+2 L$.

This shows that $v(x, \eta)$ satisfies the condition (BS). The theorem is proved.

Therefore it is sufficient to prove Lemma 3.1.

Proof of Lemma 3.1. It is proved by induction. In the case $n=0$, we can obtain the explicit form of $\widetilde{w}_{0}(x, \eta, t)$ :

$$
\widetilde{w}_{0}(x, \eta, t)=f(x-\mathrm{a} \eta, 0)+\int_{0}^{t} h(x-\mathrm{a}(\eta-s), s) d s .
$$

Therefore from the condition, it is easy to prove that $\widetilde{w}_{0}(x, \eta, t)$ is well-defined and holomorphic on $\left\{(x, \eta, t) \in \mathbf{C}^{3} ;|x| \leq r, \eta \in E_{+}\left(\theta, \rho-\varepsilon_{0}\right), t \in G_{\eta}^{\varepsilon_{0}}\right\}$ and has the estimate

$$
\left|\widetilde{w}_{0}\left(x, \eta, G_{\eta}(R)\right)\right| \leq C_{1} e^{\delta_{1}|\eta|}
$$

on $\left\{(x, \eta, t) \in \mathbf{C}^{3} ;|x| \leq r, \eta \in E_{+}\left(\theta, \rho-\varepsilon_{0}\right), t \in G_{\eta}\right\}$ for some positive constants $C_{1}$ and $\delta_{1}$. This implies the lemma for $n=0$. Next, let us assume that the lemma is proved up to $n$. Since $\left\{w_{n}(x, \eta)\right\}_{n=0}^{\infty}$ is determined by

$$
w_{n+1}(x, \eta)=\frac{\mathrm{c}}{\mathrm{a}} \int_{0}^{\eta} w_{n}(x-\mathrm{a}(\eta-t), t) d t-\frac{\mathrm{c}}{\mathrm{a}} \int_{0}^{\eta} w_{n}(x, t) d t
$$

we have 


$$
\begin{aligned}
& \widetilde{w}_{n+1}(x, \eta, t) \\
& =w_{n+1}(x-\mathrm{a}(\eta-t), t) \\
& =\frac{\mathrm{c}}{\mathrm{a}} \int_{0}^{t} w_{n}(x-\mathrm{a}(\eta-t)-\mathrm{a}(t-s), s) d s-\frac{\mathrm{c}}{\mathrm{a}} \int_{0}^{t} w_{n}(x-\mathrm{a}(\eta-t), s) d s \\
& =\frac{\mathrm{c}}{\mathrm{a}} \int_{0}^{t} w_{n}(x-\mathrm{a}(\eta-s), s) d s-\frac{\mathrm{c}}{\mathrm{a}} \int_{0}^{t} w_{n}(x-\mathrm{a}\{(\eta-t+s)-s\}, s) d s \\
& =\frac{\mathrm{c}}{\mathrm{a}} \int_{0}^{t} \widetilde{w}_{n}(x, \eta, s) d s-\frac{\mathrm{c}}{\mathrm{a}} \int_{0}^{t} \widetilde{w}_{n}(x, \eta-t+s, s) d s \\
& =: I_{1}(x, \eta, t)+I_{2}(x, \eta, t) .
\end{aligned}
$$

Let us prove that each $I_{i}(x, \eta, t)$ is well-defined on $\left\{(x, \eta, t) \in \mathbf{C}^{3} ;|x| \leq\right.$ $\left.r, \eta \in E_{+}\left(\theta, \rho-\sum_{j=0}^{n+1} \varepsilon_{j}\right), t \in G_{\eta}^{\varepsilon_{n+1}}\right\}$. We put $t=G_{\eta}(\tau)\left(\tau \in U_{\varepsilon_{n+1}}[0,|\eta|]\right)$.

On $I_{1}\left(x, \eta, G_{\eta}(\tau)\right)$ : It is clear that $\eta \in E_{+}\left(\theta, \rho-\sum_{j=0}^{n+1} \varepsilon_{j}\right) \subset E_{+}(\theta, \rho-$ $\left.\sum_{j=0}^{n} \varepsilon_{j}\right)$. By taking an integral path as

$$
s(\sigma)=G_{\eta}(\sigma)(\sigma \in[0, \tau])
$$

where $[0, \tau]$ is a segment from 0 to $\tau$, it holds that $s(\sigma) \in G_{\eta}^{\varepsilon_{n+1}} \subset G_{\eta}^{\varepsilon_{n}}$. Hence $\widetilde{w}_{n}(x, \eta, s(\sigma))$ is well-defined and $I_{1}\left(x, \eta, G_{\eta}(\tau)\right)$ is well-defined.

On $I_{2}\left(x, \eta, G_{\eta}(\tau)\right)$ : By taking an integral path as $s(\sigma)=G_{\eta}(\sigma)(\sigma \in[0, \tau])$, it holds that $\eta-G_{\eta}(\tau)+s(\sigma) \in E_{+}\left(\theta, \rho-\sum_{j=0}^{n} \varepsilon_{j}\right)$ and $s(\sigma) \in G_{\eta-G_{\eta}(\tau)+s(\sigma)}^{\varepsilon_{n+1}} \subset$ $G_{\eta-G_{\eta}(\tau)+s(\sigma)}^{\varepsilon_{n}}$. Hence $\widetilde{w}_{n}\left(x, \eta-G_{\eta}(\tau)+s(\sigma), s(\sigma)\right)$ is well-defined and $I_{2}(x, \eta$, $\left.G_{\eta}(\tau)\right)$ is well-defined.

Therefore $\widetilde{w}_{n+1}(x, \eta, t)$ is well-defined and holomorphic on $\{(x, \eta, t) \in$ $\left.\mathbf{C}^{3} ;|x| \leq r, \eta \in E_{+}\left(\theta, \rho-\sum_{j=0}^{n+1} \varepsilon_{j}\right), t \in G_{\eta}^{\varepsilon_{n+1}}\right\}$. Moreover on $\{(x, \eta, t) \in$ $\left.\mathbf{C}^{3} ;|x| \leq r, \eta \in E_{+}\left(\theta, \rho-\sum_{j=0}^{n+1} \varepsilon_{j}\right), t \in G_{\eta}\right\}$ we have the following representations:

$$
\begin{aligned}
& I_{1}\left(x, \eta, G_{\eta}(R)\right)=\frac{\mathrm{c}}{\mathrm{a}} \int_{0}^{R} \widetilde{w}_{n}\left(x, \eta, G_{\eta}\left(R_{1}\right)\right) e^{i \arg (\eta)} d R_{1}, \\
& I_{2}\left(x, \eta, G_{\eta}(R)\right)=-\frac{\mathrm{c}}{\mathrm{a}} \int_{0}^{R} \widetilde{w}_{n}\left(x,\left(|\eta|-R+R_{1}\right) e^{i \arg (\eta)},\right.
\end{aligned}
$$

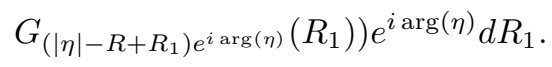

Let us estimate each $I_{i}\left(x, \eta, G_{\eta}(R)\right)$.

On $I_{1}\left(x, \eta, G_{\eta}(R)\right)$ : It follows from the assumption of the induction that

$$
\left|\widetilde{w}_{n}\left(x, \eta, G_{\eta}\left(R_{1}\right)\right)\right| \leq C_{1} e^{\delta_{1}|\eta|} L^{n} \sum_{k=0}^{n}\left(\begin{array}{l}
n \\
k
\end{array}\right) \frac{1}{\delta_{1}^{n-k}} \frac{R_{1}^{k}}{k !},
$$


which implies that

$$
\begin{aligned}
\left|I_{1}\left(x, \eta, G_{\eta}(R)\right)\right| & \leq C_{1} e^{\delta_{1}|\eta|} L^{n+1} \sum_{k=0}^{n}\left(\begin{array}{l}
n \\
k
\end{array}\right) \frac{1}{\delta_{1}^{n-k}} \int_{0}^{R} \frac{R_{1}^{k}}{k !} d R_{1} \\
& =C_{1} e^{\delta_{1}|\eta|} L^{n+1} \sum_{k=0}^{n}\left(\begin{array}{l}
n \\
k
\end{array}\right) \frac{1}{\delta_{1}^{n-k}} \frac{R^{k+1}}{(k+1) !} .
\end{aligned}
$$

On $I_{2}\left(x, \eta, G_{\eta}(R)\right)$ : By the assumption of the induction, we have

$$
\begin{gathered}
\mid \widetilde{w}_{n}\left(x,\left(|\eta|-R+R_{1}\right) e^{i \arg (\eta)}, G_{\left.\left(|\eta|-R+R_{1}\right) e^{i \arg (\eta)}\left(R_{1}\right)\right) \mid}\right. \\
\leq C_{1} e^{\delta_{1}|\eta|} e^{-\delta_{1} R} e^{\delta_{1} R_{1}} L^{n} \sum_{k=0}^{n}\left(\begin{array}{l}
n \\
k
\end{array}\right) \frac{1}{\delta_{1}^{n-k}} \frac{R_{1}^{k}}{k !}
\end{gathered}
$$

which implies that

$$
\left|I_{2}\left(x, \eta, G_{\eta}(R)\right)\right| \leq C_{1} e^{\delta_{1}|\eta|} e^{-\delta_{1} R} L^{n+1} \sum_{k=0}^{n}\left(\begin{array}{l}
n \\
k
\end{array}\right) \frac{1}{\delta_{1}^{n-k}} \int_{0}^{R} e^{\delta_{1} R_{1}} \frac{R_{1}^{k}}{k !} d R_{1} .
$$

Here it holds that

$$
\begin{aligned}
\int_{0}^{R} e^{\delta_{1} R_{1}} \frac{R_{1}^{k}}{k !} d R_{1} & =\int_{0}^{R}\left\{\frac{d}{d R_{1}}\left\{\frac{1}{\delta_{1}} e^{\delta_{1} R_{1}}\right\}\right\} \frac{R_{1}^{k}}{k !} d R_{1} \\
& \leq\left[\frac{1}{\delta_{1}} e^{\delta_{1} R_{1}} \frac{R_{1}^{k}}{k !}\right]_{R_{1}=0}^{R} \\
& \leq \frac{1}{\delta_{1}} e^{\delta_{1} R} \frac{R^{k}}{k !}
\end{aligned}
$$

Hence we obtain

$$
\left|I_{2}\left(x, \eta, G_{\eta}(R)\right)\right| \leq C_{1} e^{\delta_{1}|\eta|} L^{n+1} \sum_{k=0}^{n}\left(\begin{array}{l}
n \\
k
\end{array}\right) \frac{1}{\delta_{1}^{n+1-k}} \frac{R^{k}}{k !} .
$$

Therefore it holds that

$$
\begin{aligned}
& \left|\widetilde{w}_{n+1}\left(x, \eta, G_{\eta}(R)\right)\right| \\
& \leq C_{1} e^{\delta_{1}|\eta|} L^{n+1}\left\{\sum_{k=0}^{n}\left(\begin{array}{l}
n \\
k
\end{array}\right) \frac{1}{\delta_{1}^{n-k}} \frac{R^{k+1}}{(k+1) !}+\sum_{k=0}^{n}\left(\begin{array}{l}
n \\
k
\end{array}\right) \frac{1}{\delta_{1}^{n+1-k}} \frac{R^{k}}{k !}\right\} \\
& =C_{1} e^{\delta_{1}|\eta|} L^{n+1}\left\{\sum_{k=1}^{n+1}\left(\begin{array}{c}
n \\
k-1
\end{array}\right) \frac{1}{\delta_{1}^{n+1-k}} \frac{R^{k}}{k !}+\sum_{k=0}^{n}\left(\begin{array}{l}
n \\
k
\end{array}\right) \frac{1}{\delta_{1}^{n+1-k}} \frac{R^{k}}{k !}\right\} \\
& =C_{1} e^{\delta_{1}|\eta|} L^{n+1}\left[\frac{1}{\delta_{1}^{n+1}}+\sum_{k=1}^{n}\left\{\left(\begin{array}{c}
n \\
k-1
\end{array}\right)+\left(\begin{array}{l}
n \\
k
\end{array}\right)\right\} \frac{1}{\delta_{1}^{n+1-k}} \frac{R^{k}}{k !}+\frac{R^{n+1}}{(n+1) !}\right]
\end{aligned}
$$




$$
\begin{aligned}
& =C_{1} e^{\delta_{1}|\eta|} L^{n+1}\left\{\frac{1}{\delta_{1}^{n+1}}+\sum_{k=1}^{n}\left(\begin{array}{c}
n+1 \\
k
\end{array}\right) \frac{1}{\delta_{1}^{n+1-k}} \frac{R^{k}}{k !}+\frac{R^{n+1}}{(n+1) !}\right\} \\
& =C_{1} e^{\delta_{1}|\eta|} L^{n+1} \sum_{k=0}^{n+1}\left(\begin{array}{c}
n+1 \\
k
\end{array}\right) \frac{1}{\delta_{1}^{n+1-k}} \frac{R^{k}}{k !},
\end{aligned}
$$

which implies the lemma for $n+1$. The proof is completed.

\section{§4. The Proof of Theorem 1.2 (Case (2))}

In Case $(2)(b=0$ and $d \neq 0)$, the equation (2.5) is written as follows:

$$
\begin{aligned}
\left\{(1+\mathrm{d} \eta) D_{\eta}+\mathrm{a} D_{x}\right\} v(x, \eta) & =-\mathrm{c} D_{\eta}^{-1} D_{x} v(x, \eta)+h(x, \eta), \\
v(x, 0) & =f(x, 0)
\end{aligned}
$$

We will prove that the solution $v(x, \eta)$ of $(4.1)$ satisfies the condition (BS) in Theorem 2.1. First, we remark that in general the solution $w(x, \eta)$ of the initial value problem of the following first order linear partial differential equation

$$
\begin{gathered}
\left\{(1+\mathrm{d} \eta) D_{\eta}+\mathrm{a} D_{x}\right\} w(x, \eta)=k(x, \eta) \\
w(x, 0)=l(x)
\end{gathered}
$$

is given by

$$
\begin{aligned}
w(x, \eta)= & l\left(x-\frac{\mathrm{a}}{\mathrm{d}} \log (1+\mathrm{d} \eta)\right) \\
& +\int_{0}^{\eta} k\left(x-\frac{\mathrm{a}}{\mathrm{d}} \log \frac{1+\mathrm{d} \eta}{1+\mathrm{d} t}, t\right) \frac{1}{1+\mathrm{d} t} d t .
\end{aligned}
$$

Proof of the theorem. We assume that $c \neq 0$. The equation (4.1) is rewritten as follows:

$$
\begin{aligned}
\left\{(1+\mathrm{d} \eta) D_{\eta}+\mathrm{a} D_{x}\right\} v(x, \eta) & =-\mathrm{c} \int_{0}^{\eta} v_{x}(x, s) d s+h(x, \eta), \\
v(x, 0) & =f(x, 0) .
\end{aligned}
$$

Let us transform (4.4) into the integral equation. It follows from (4.3) that the equation (4.4) is equivalent to the following equation:

$$
\begin{aligned}
v(x, \eta)= & f\left(x-\frac{\mathrm{a}}{\mathrm{d}} \log (1+\mathrm{d} \eta), 0\right)+\int_{0}^{\eta} h\left(x-\frac{\mathrm{a}}{\mathrm{d}} \log \frac{1+\mathrm{d} \eta}{1+\mathrm{d} t}, t\right) \frac{1}{1+\mathrm{d} t} d t \\
& -\mathrm{c} \int_{0}^{\eta} \int_{0}^{t} v_{x}\left(x-\frac{\mathrm{a}}{\mathrm{d}} \log \frac{1+\mathrm{d} \eta}{1+\mathrm{d} t}, s\right) \frac{1}{1+\mathrm{d} t} d s d t .
\end{aligned}
$$


Here we remark that

$$
\begin{aligned}
& \int_{0}^{\eta} \int_{0}^{t} v_{x}\left(x-\frac{\mathrm{a}}{\mathrm{d}} \log \frac{1+\mathrm{d} \eta}{1+\mathrm{d} t}, s\right) \frac{1}{1+\mathrm{d} t} d s d t \\
& =\int_{0}^{\eta} \int_{s}^{\eta} v_{x}\left(x-\frac{\mathrm{a}}{\mathrm{d}} \log \frac{1+\mathrm{d} \eta}{1+\mathrm{d} t}, s\right) \frac{1}{1+\mathrm{d} t} d t d s \\
& =\int_{0}^{\eta} \int_{s}^{\eta} \frac{d}{d t}\left\{\frac{1}{\mathrm{a}} v\left(x-\frac{\mathrm{a}}{\mathrm{d}} \log \frac{1+\mathrm{d} \eta}{1+\mathrm{d} t}, s\right)\right\} d t d s \\
& =\frac{1}{\mathrm{a}} \int_{0}^{\eta} v(x, t) d t-\frac{1}{\mathrm{a}} \int_{0}^{\eta} v\left(x-\frac{\mathrm{a}}{\mathrm{d}} \log \frac{1+\mathrm{d} \eta}{1+\mathrm{d} t}, t\right) d t
\end{aligned}
$$

Therefore we know that (4.4) is equivalent to the following integral equation:

$$
\begin{aligned}
v(x, \eta)= & f\left(x-\frac{\mathrm{a}}{\mathrm{d}} \log (1+\mathrm{d} \eta), 0\right) \\
& +\int_{0}^{\eta} h\left(x-\frac{\mathrm{a}}{\mathrm{d}} \log \frac{1+\mathrm{d} \eta}{1+\mathrm{d} t}, t\right) \frac{1}{1+\mathrm{d} t} d t \\
& +\frac{\mathrm{c}}{\mathrm{a}} \int_{0}^{\eta} v\left(x-\frac{\mathrm{a}}{\mathrm{d}} \log \frac{1+\mathrm{d} \eta}{1+\mathrm{d} t}, t\right) d t-\frac{\mathrm{c}}{\mathrm{a}} \int_{0}^{\eta} v(x, t) d t .
\end{aligned}
$$

In order to prove that the solution $v(x, \eta)$ of $(4.5)$ satisfies the condition (BS), we employ the iteration method. Let us define $\left\{v_{n}(x, \eta)\right\}_{n=0}^{\infty}$ as follows:

$$
v_{0}(x, \eta)=f\left(x-\frac{\mathrm{a}}{\mathrm{d}} \log (1+\mathrm{d} \eta), 0\right)+\int_{0}^{\eta} h\left(x-\frac{\mathrm{a}}{\mathrm{d}} \log \frac{1+\mathrm{d} \eta}{1+\mathrm{d} t}, t\right) \frac{1}{1+\mathrm{d} t} d t .
$$

For $n \geq 0$,

$$
v_{n+1}(x, \eta)=v_{0}(x, \eta)+\frac{\mathrm{c}}{\mathrm{a}} \int_{0}^{\eta} v_{n}\left(x-\frac{\mathrm{a}}{\mathrm{d}} \log \frac{1+\mathrm{d} \eta}{1+\mathrm{d} t}, t\right) d t-\frac{\mathrm{c}}{\mathrm{a}} \int_{0}^{\eta} v_{n}(x, t) d t .
$$

Next we put $w_{0}(x, \eta):=v_{0}(x, \eta)$ and $w_{n}(x, \eta):=v_{n}(x, \eta)-v_{n-1}(x, \eta)$ for $n \geq 1$, and we define $\widetilde{w}_{n}(x, \eta, t)$ by

$$
\widetilde{w}_{n}(x, \eta, t):=w_{n}\left(x-\frac{\mathrm{a}}{\mathrm{d}} \log \frac{1+\mathrm{d} \eta}{1+\mathrm{d} t}, t\right) .
$$

Definition 4.1. For $\eta \in \mathbf{C}$, we define a function $\widetilde{G}_{\eta}(\tau)$ by

$$
\widetilde{G}_{\eta}(\tau)=\frac{\tau e^{i \arg (\eta)}}{1+\mathrm{d}(|\eta|-\tau) e^{i \arg (\eta)}}, \quad \tau \in \mathbf{C}
$$


and put

$$
\begin{aligned}
& \widetilde{G}_{\eta}:=\left\{\widetilde{G}_{\eta}(R) \in \mathbf{C} ; 0 \leq R \leq|\eta|\right\}, \\
& \widetilde{G}_{\eta}^{\varepsilon}:=\widetilde{G}_{\eta}\left(U_{\varepsilon}[0,|\eta|]\right) .
\end{aligned}
$$

Now let us take a monotonically decreasing positive sequence $\left\{\varepsilon_{n}\right\}_{n=0}^{\infty}$ satisfying (3.8). Furthermore let us take $K>1$ so that

$$
K^{-1} \frac{1}{1+|\eta|} \leq\left|\frac{1}{1+\mathrm{d} \eta}\right| \leq K \frac{1}{1+|\eta|}
$$

for $\eta \in E_{+}(\theta, \rho)$. Then we obtain the following lemma:

Lemma 4.1. $\widetilde{w}_{n}(x, \eta, t)$ is continued analytically to $\left\{(x, \eta, t) \in \mathbf{C}^{3} ;|x|\right.$ $\left.\leq r, \eta \in E_{+}\left(\theta, \rho-\sum_{j=0}^{n} \varepsilon_{j}\right), t \in \widetilde{G}_{\eta}^{\varepsilon_{n}}\right\}$. Furthermore on $\left\{(x, \eta, t) \in \mathbf{C}^{3} ;|x| \leq\right.$ $\left.r, \eta \in E_{+}\left(\theta, \rho-\sum_{j=0}^{n} \varepsilon_{j}\right), t \in \widetilde{G}_{\eta}\right\}$ we have the following estimate: For some positive constants $C_{1}$ and $\delta_{1}$,

$$
\left|\widetilde{w}_{n}\left(x, \eta, \widetilde{G}_{\eta}(R)\right)\right| \leq C_{1} e^{\delta_{1}|\eta|}\left(L K^{3}\right)^{n} \frac{1}{(1+|\eta|-R)^{n}} \sum_{k=0}^{n}\left(\begin{array}{l}
n \\
k
\end{array}\right) \frac{1}{\delta_{1}^{n-k}} \frac{(1+|\eta|)^{k}}{k !} .
$$

If we admit Lemma 4.1, the theorem is proved similarly to Case (1).

Proof of Lemma 4.1. It is proved by the induction. We omit the proof for $n=0$, since it is proved directly by using the explicit expression of $\widetilde{w}_{0}(x, \eta, t)$ as mentioned in the proof of Lemma 3.1. Let us assume that the lemma is proved up to $n$. Since $\left\{w_{n}(x, \eta)\right\}_{n=0}^{\infty}$ is determined by

$$
w_{n+1}(x, \eta)=\frac{\mathrm{c}}{\mathrm{a}} \int_{0}^{\eta} w_{n}\left(x-\frac{\mathrm{a}}{\mathrm{d}} \log \frac{1+\mathrm{d} \eta}{1+\mathrm{d} t}, t\right) d t-\frac{\mathrm{c}}{\mathrm{a}} \int_{0}^{\eta} w_{n}(x, t) d t,
$$

we have

$$
\begin{aligned}
\widetilde{w}_{n+1}(x, \eta, t)= & w_{n+1}\left(x-\frac{\mathrm{a}}{\mathrm{d}} \log \frac{1+\mathrm{d} \eta}{1+\mathrm{d} t}, t\right) \\
= & \frac{\mathrm{c}}{\mathrm{a}} \int_{0}^{t} w_{n}\left(x-\frac{\mathrm{a}}{\mathrm{d}} \log \frac{1+\mathrm{d} \eta}{1+\mathrm{d} t}-\frac{\mathrm{a}}{\mathrm{d}} \log \frac{1+\mathrm{d} t}{1+\mathrm{d} s}, s\right) d s \\
& -\frac{\mathrm{c}}{\mathrm{a}} \int_{0}^{t} w_{n}\left(x-\frac{\mathrm{a}}{\mathrm{d}} \log \frac{1+\mathrm{d} \eta}{1+\mathrm{d} t}, s\right) d s
\end{aligned}
$$




$$
\begin{aligned}
= & \frac{\mathrm{c}}{\mathrm{a}} \int_{0}^{t} w_{n}\left(x-\frac{\mathrm{a}}{\mathrm{d}} \log \frac{1+\mathrm{d} \eta}{1+\mathrm{d} s}, s\right) d s \\
& -\frac{\mathrm{c}}{\mathrm{a}} \int_{0}^{t} w_{n}\left(x-\frac{\mathrm{a}}{\mathrm{d}} \log \left(\frac{1+\mathrm{d} \eta}{1+\mathrm{d} t} \frac{1+\mathrm{d} s}{1+\mathrm{d} s}\right), s\right) d s \\
= & \frac{\mathrm{c}}{\mathrm{a}} \int_{0}^{t} \widetilde{w}_{n}(x, \eta, s) d s-\frac{\mathrm{c}}{\mathrm{a}} \int_{0}^{t} \widetilde{w}_{n}(x, \zeta(\eta, t, s), s) d s \\
= & : I_{1}(x, \eta, t)+I_{2}(x, \eta, t),
\end{aligned}
$$

where $\zeta(\eta, t, s)$ is defined by

$$
\frac{(1+\mathrm{d} \eta)(1+\mathrm{d} s)}{1+\mathrm{d} t}=1+\mathrm{d} \zeta(\eta, t, s)
$$

Let us prove that each $I_{i}(x, \eta, t)$ is well-defined on $\left\{(x, \eta, t) \in \mathbf{C}^{3} ;|x| \leq\right.$ $\left.r, \eta \in E_{+}\left(\theta, \rho-\sum_{j=0}^{n+1} \varepsilon_{j}\right), t \in \widetilde{G}_{\eta}^{\varepsilon_{n+1}}\right\}$. We put $t=\widetilde{G}_{\eta}(\tau)\left(\tau \in U_{\varepsilon_{n+1}}[0,|\eta|]\right)$.

On $I_{1}\left(x, \eta, \widetilde{G}_{\eta}(\tau)\right)$ : It is clear that $\eta \in E_{+}\left(\theta, \rho-\sum_{j=0}^{n+1} \varepsilon_{j}\right) \subset E_{+}(\theta, \rho-$ $\left.\sum_{j=0}^{n} \varepsilon_{j}\right)$. By taking an integral path as

$$
s(\sigma)=\widetilde{G}_{\eta}(\sigma)=\frac{\sigma e^{i \arg (\eta)}}{1+\mathrm{d}(|\eta|-\sigma) e^{i \arg (\eta)}}(\sigma \in[0, \tau]),
$$

it holds that $s(\sigma) \in \widetilde{G}_{\eta}^{\varepsilon_{n+1}} \subset \widetilde{G}_{\eta}^{\varepsilon_{n}}$. Hence $\widetilde{w}_{n}(x, \eta, s(\sigma))$ is well-defined and $I_{1}\left(x, \eta, \widetilde{G}_{\eta}(\tau)\right)$ is well-defined.

On $I_{2}\left(x, \eta, \widetilde{G}_{\eta}(\tau)\right)$ : By taking an integral path as

$$
s(\sigma)=\frac{\sigma e^{i \arg (\eta)}}{1+\mathrm{d}(|\eta|-\tau) e^{i \arg (\eta)}}(\sigma \in[0, \tau]),
$$

we have $\zeta\left(\eta, \widetilde{G}_{\eta}(\tau), s(\sigma)\right) \in E_{+}\left(\theta, \rho-\sum_{j=0}^{n} \varepsilon_{j}\right)$ and $s(\sigma) \in \widetilde{G}_{\zeta\left(\eta, \widetilde{G}_{\eta}(\tau), s(\sigma)\right)}^{\varepsilon_{n+1}} \subset$ $\widetilde{G}_{\zeta\left(\eta, \widetilde{G}_{\eta}(\tau), s(\sigma)\right)}^{\varepsilon_{n}}$. Hence $\widetilde{w}_{n}\left(x, \eta-\widetilde{G}_{\eta}(\tau)+s(\sigma), s(\sigma)\right)$ is well-defined and $I_{2}(x, \eta$, $\left.\widetilde{G}_{\eta}(\tau)\right)$ is well-defined.

Therefore $\widetilde{w}_{n+1}(x, \eta, t)$ is well-defined and holomorphic on $\{(x, \eta, t) \in$ $\left.\mathbf{C}^{3} ;|x| \leq r, \eta \in E_{+}\left(\theta, \rho-\sum_{j=0}^{n+1} \varepsilon_{j}\right), t \in \widetilde{G}_{\eta}^{\varepsilon_{n+1}}\right\}$. Moreover on $\{(x, \eta, t) \in$ $\left.\mathbf{C}^{3} ;|x| \leq r, \eta \in E_{+}\left(\theta, \rho-\sum_{j=0}^{n+1} \varepsilon_{j}\right), t \in \widetilde{G}_{\eta}\right\}$ we have the following representations:

$$
\begin{aligned}
& I_{1}\left(x, \eta, \widetilde{G}_{\eta}(R)\right)= \frac{\mathrm{c}}{\mathrm{a}} \int_{0}^{R} \widetilde{w}_{n}\left(x, \eta, \widetilde{G}_{\eta}\left(R_{1}\right)\right) \frac{(1+\mathrm{d} \eta) e^{i \arg (\eta)}}{\left\{1+\mathrm{d}\left(|\eta|-R_{1}\right) e^{i \arg (\eta)}\right\}^{2}} d R_{1}, \\
& I_{2}\left(x, \eta, \widetilde{G}_{\eta}(R)\right)= \frac{\mathrm{c}}{\mathrm{a}} \frac{1}{1+\mathrm{d}(|\eta|-R) e^{i \arg (\eta)}} \\
& \times \int_{0}^{R} \widetilde{w}_{n}\left(x,\left(|\eta|-R+R_{1}\right) e^{i \arg (\eta)},\right. \\
& \widetilde{G}_{\left.\left(|\eta|-R+R_{1}\right) e^{i \arg (\eta)}\left(R_{1}\right)\right) e^{i \arg (\eta)} d R_{1} .}
\end{aligned}
$$


Let us estimate each $I_{i}\left(x, \eta, \widetilde{G}_{\eta}(R)\right)$.

On $I_{1}\left(x, \eta, \widetilde{G}_{\eta}(R)\right)$ : It follows from the assumption of the induction that

$$
\left|\widetilde{w}_{n}\left(x, \eta, \widetilde{G}_{\eta}\left(R_{1}\right)\right)\right| \leq C_{1} e^{\delta_{1}|\eta|}\left(L K^{3}\right)^{n} \frac{1}{\left(1+|\eta|-R_{1}\right)^{n}} \sum_{k=0}^{n}\left(\begin{array}{l}
n \\
k
\end{array}\right) \frac{1}{\delta_{1}^{n-k}} \frac{(1+|\eta|)^{k}}{k !}
$$

which implies that

$$
\begin{aligned}
&\left|I_{1}\left(x, \eta, \widetilde{G}_{\eta}(R)\right)\right| \\
& \leq L \int_{0}^{R} C_{1} e^{\delta_{1}|\eta|}\left(L K^{3}\right)^{n} \frac{1}{\left(1+|\eta|-R_{1}\right)^{n}} \\
& \times \sum_{k=0}^{n}\left(\begin{array}{l}
n \\
k
\end{array}\right) \frac{1}{\delta_{1}^{n-k}} \frac{(1+|\eta|)^{k}}{k !} K^{3} \frac{1+|\eta|}{\left(1+|\eta|-R_{1}\right)^{2}} d R_{1} \\
&= C_{1} e^{\delta_{1}|\eta|}\left(L K^{3}\right)^{n+1} \\
& \times \sum_{k=0}^{n}\left(\begin{array}{l}
n \\
k
\end{array}\right) \frac{1}{\delta_{1}^{n-k}} \frac{(1+|\eta|)^{k+1}}{k !} \int_{0}^{R} \frac{1}{\left(1+|\eta|-R_{1}\right)^{n+2}} d R_{1} \\
& \leq C_{1} e^{\delta_{1}|\eta|}\left(L K^{3}\right)^{n+1} \sum_{k=0}^{n}\left(\begin{array}{l}
n \\
k
\end{array}\right) \frac{1}{\delta_{1}^{n-k}} \frac{(1+|\eta|)^{k+1}}{k !} \frac{1}{n+1} \frac{1}{(1+|\eta|-R)^{n+1}} \\
& \leq C_{1} e^{\delta_{1}|\eta|}\left(L K^{3}\right)^{n+1} \frac{1}{(1+|\eta|-R)^{n+1}} \sum_{k=0}^{n}\left(\begin{array}{l}
n \\
k
\end{array}\right) \frac{1}{\delta_{1}^{n-k}} \frac{(1+|\eta|)^{k+1}}{(k+1) !} .
\end{aligned}
$$

On $I_{2}\left(x, \eta, \widetilde{G}_{\eta}(R)\right)$ : By the assumption of the induction, we have

$$
\begin{aligned}
\mid \widetilde{w}_{n}( & x,\left(|\eta|-R+R_{1}\right) e^{i \arg (\eta)}, \widetilde{G}_{\left.\left(|\eta|-R+R_{1}\right) e^{i \arg (\eta)}\left(R_{1}\right)\right) \mid} \\
\leq & C_{1} e^{\delta_{1}|\eta|} e^{-\delta_{1} R} e^{\delta_{1} R_{1}}\left(L K^{3}\right)^{n} \frac{1}{\left(1+|\eta|-R+R_{1}-R_{1}\right)^{n}} \\
& \times \sum_{k=0}^{n}\left(\begin{array}{l}
n \\
k
\end{array}\right) \frac{1}{\delta_{1}^{n-k}} \frac{\left(1+|\eta|-R+R_{1}\right)^{k}}{k !} \\
= & C_{1} e^{\delta_{1}|\eta|} e^{-\delta_{1} R} e^{\delta_{1} R_{1}}\left(L K^{3}\right)^{n} \frac{1}{(1+|\eta|-R)^{n}} \\
& \times \sum_{k=0}^{n}\left(\begin{array}{l}
n \\
k
\end{array}\right) \frac{1}{\delta_{1}^{n-k}} \frac{\left(1+|\eta|-R+R_{1}\right)^{k}}{k !},
\end{aligned}
$$

which implies that

$$
\begin{aligned}
& \left|I_{2}\left(x, \eta, \widetilde{G}_{\eta}(R)\right)\right| \\
& \quad \leq L K \frac{1}{1+|\eta|-R} \int_{0}^{R} C_{1} e^{\delta_{1}|\eta|} e^{-\delta_{1} R} e^{\delta_{1} R_{1}}\left(L K^{3}\right)^{n} \frac{1}{(1+|\eta|-R)^{n}}
\end{aligned}
$$




$$
\begin{aligned}
& \times \sum_{k=0}^{n}\left(\begin{array}{l}
n \\
k
\end{array}\right) \frac{1}{\delta_{1}^{n-k}} \frac{\left(1+|\eta|-R+R_{1}\right)^{k}}{k !} d R_{1} \\
\leq & C_{1} e^{\delta_{1}|\eta|}\left(L K^{3}\right)^{n+1} \frac{1}{(1+|\eta|-R)^{n+1}} \\
& \times \sum_{k=0}^{n}\left(\begin{array}{l}
n \\
k
\end{array}\right) \frac{1}{\delta_{1}^{n-k}} e^{-\delta_{1} R} \int_{0}^{R} e^{\delta_{1} R_{1}} \frac{\left(1+|\eta|-R+R_{1}\right)^{k}}{k !} d R_{1} .
\end{aligned}
$$

Here it holds that

$$
\begin{aligned}
\int_{0}^{R} & e^{\delta_{1} R_{1}} \frac{\left(1+|\eta|-R+R_{1}\right)^{k}}{k !} d R_{1} \\
& =\int_{0}^{R} \frac{d}{d R_{1}}\left\{\frac{1}{\delta_{1}} e^{\delta_{1} R_{1}}\right\} \frac{\left(1+|\eta|-R+R_{1}\right)^{k}}{k !} d R_{1} \\
& \leq\left[\frac{1}{\delta_{1}} e^{\delta_{1} R_{1}} \frac{\left(1+|\eta|-R+R_{1}\right)^{k}}{k !}\right]_{R_{1}=0}^{R} \\
& \leq \frac{1}{\delta_{1}} e^{\delta_{1} R} \frac{(1+|\eta|)^{k}}{k !}
\end{aligned}
$$

Hence we obtain

$$
\begin{aligned}
& \left|I_{2}\left(x, \eta, \widetilde{G}_{\eta}(R)\right)\right| \\
& \quad \leq C_{1} e^{\delta_{1}|\eta|}\left(L K^{3}\right)^{n+1} \frac{1}{(1+|\eta|-R)^{n+1}} \sum_{k=0}^{n}\left(\begin{array}{l}
n \\
k
\end{array}\right) \frac{1}{\delta_{1}^{n+1-k}} \frac{(1+|\eta|)^{k}}{k !} .
\end{aligned}
$$

Therefore it holds that

$$
\begin{aligned}
&\left|\widetilde{w}_{n+1}\left(x, \eta, \widetilde{G}_{\eta}(R)\right)\right| \\
& \leq C_{1} e^{\delta_{1}|\eta|}\left(L K^{3}\right)^{n+1} \frac{1}{(1+|\eta|-R)^{n+1}} \\
& \times\left\{\sum_{k=0}^{n}\left(\begin{array}{l}
n \\
k
\end{array}\right) \frac{1}{\delta_{1}^{n-k}} \frac{(1+|\eta|)^{k+1}}{(k+1) !}+\sum_{k=0}^{n}\left(\begin{array}{l}
n \\
k
\end{array}\right) \frac{1}{\delta_{1}^{n+1-k}} \frac{(1+|\eta|)^{k}}{k !}\right\} \\
&= C_{1} e^{\delta_{1}|\eta|}\left(L K^{3}\right)^{n+1} \frac{1}{(1+|\eta|-R)^{n+1}} \\
& \times\left\{\sum_{k=1}^{n+1}\left(\begin{array}{c}
n \\
k-1
\end{array}\right) \frac{1}{\delta_{1}^{n+1-k}} \frac{(1+|\eta|)^{k}}{k !}+\sum_{k=0}^{n}\left(\begin{array}{l}
n \\
k
\end{array}\right) \frac{1}{\delta_{1}^{n+1-k}} \frac{(1+|\eta|)^{k}}{k !}\right\} \\
&= C_{1} e^{\delta_{1}|\eta|}\left(L K^{3}\right)^{n+1} \frac{1}{(1+|\eta|-R)^{n+1}} \\
& \times\left[\frac{1}{\delta_{1}^{n+1}}+\sum_{k=1}^{n}\left\{\left(\begin{array}{c}
n \\
k-1
\end{array}\right)+\left(\begin{array}{l}
n \\
k
\end{array}\right)\right\} \frac{1}{\delta_{1}^{n+1-k}} \frac{(1+|\eta|)^{k}}{k !}+\frac{(1+|\eta|)^{n+1}}{(n+1) !}\right]
\end{aligned}
$$




$$
=C_{1} e^{\delta_{1}|\eta|}\left(L K^{3}\right)^{n+1} \frac{1}{(1+|\eta|-R)^{n+1}} \sum_{k=0}^{n+1}\left(\begin{array}{c}
n+1 \\
k
\end{array}\right) \frac{1}{\delta_{1}^{n+1-k}} \frac{(1+|\eta|)^{k}}{k !},
$$

which implies the lemma for $n+1$. The proof is completed.

\section{$\S 5 . \quad$ The Proof of Theorem 1.2 (Case (3))}

In Case (3) $(b \neq 0$ and $d=0)$, the equation (2.5) is written as follows:

$$
\begin{aligned}
\left\{D_{\eta}+(\mathrm{a}+\mathrm{b} x) D_{x}\right\} v(x, \eta) & =-\mathrm{c} D_{\eta}^{-1} D_{x} v(x, \eta)+h(x, \eta), \\
v(x, 0) & =f(x, 0) .
\end{aligned}
$$

We will prove that the solution $v(x, \eta)$ of (5.1) satisfies the condition (BS). First, we remark that the solution $w(x, \eta)$ of the initial value problem of the following first order linear partial differential equation

$$
\begin{gathered}
\left\{D_{\eta}+(\mathrm{a}+\mathrm{b} x) D_{x}\right\} w(x, \eta)=k(x, \eta), \\
w(x, 0)=l(x)
\end{gathered}
$$

is given by

$$
w(x, \eta)=l\left(\left(\frac{\mathrm{a}}{\mathrm{b}}+x\right) e^{-\mathrm{b} \eta}-\frac{\mathrm{a}}{\mathrm{b}}\right)+\int_{0}^{\eta} k\left(\left(\frac{\mathrm{a}}{\mathrm{b}}+x\right) e^{-\mathrm{b}(\eta-t)}-\frac{\mathrm{a}}{\mathrm{b}}, t\right) d t .
$$

Proof of the theorem. We assume that $\mathrm{c} \neq 0$. Therefore we remark that $\Re\left(-\mathrm{b} e^{i \theta}\right) \geq 0$ by the assumption (1.12). The equation (5.1) is rewritten as follows:

$$
\begin{aligned}
\left\{D_{\eta}+(\mathrm{a}+\mathrm{b} x) D_{x}\right\} v(x, \eta) & =-\mathrm{c} \int_{0}^{\eta} v_{x}(x, s) d s+h(x, \eta), \\
v(x, 0) & =f(x, 0) .
\end{aligned}
$$

Let us transform (5.4) into the integral equation. It follows from (5.3) that the equation (5.4) is equivalent to the following equation:

$$
\begin{aligned}
v(x, \eta)= & f\left(\left(\frac{\mathrm{a}}{\mathrm{b}}+x\right) e^{-\mathrm{b} \eta}-\frac{\mathrm{a}}{\mathrm{b}}, 0\right)+\int_{0}^{\eta} h\left(\left(\frac{\mathrm{a}}{\mathrm{b}}+x\right) e^{-\mathrm{b}(\eta-t)}-\frac{\mathrm{a}}{\mathrm{b}}, t\right) d t \\
& -\mathrm{c} \int_{0}^{\eta} \int_{0}^{t} v_{x}\left(\left(\frac{\mathrm{a}}{\mathrm{b}}+x\right) e^{-\mathrm{b}(\eta-t)}-\frac{\mathrm{a}}{\mathrm{b}}, s\right) d s d t .
\end{aligned}
$$


Here we remark that

$$
\begin{aligned}
\int_{0}^{\eta} & \int_{0}^{t} v_{x}\left(\left(\frac{\mathrm{a}}{\mathrm{b}}+x\right) e^{-\mathrm{b}(\eta-t)}-\frac{\mathrm{a}}{\mathrm{b}}, s\right) d s d t \\
= & \int_{0}^{\eta} \int_{s}^{\eta} v_{x}\left(\left(\frac{\mathrm{a}}{\mathrm{b}}+x\right) e^{-\mathrm{b}(\eta-t)}-\frac{\mathrm{a}}{\mathrm{b}}, s\right) d t d s \\
= & \frac{1}{(\mathrm{a}+\mathrm{b} x) e^{-\mathrm{b} \eta}} \int_{0}^{\eta} \int_{s}^{\eta} e^{-\mathrm{b} t} \frac{d}{d t} v\left(\left(\frac{\mathrm{a}}{\mathrm{b}}+x\right) e^{-\mathrm{b}(\eta-t)}-\frac{\mathrm{a}}{\mathrm{b}}, s\right) d t d s \\
= & \frac{1}{(\mathrm{a}+\mathrm{b} x) e^{-\mathrm{b} \eta}} \int_{0}^{\eta}\left\{\left[e^{-\mathrm{b} t} v\left(\left(\frac{\mathrm{a}}{\mathrm{b}}+x\right) e^{-\mathrm{b}(\eta-t)}-\frac{\mathrm{a}}{\mathrm{b}}, s\right)\right]_{t=s}^{\eta}\right. \\
= & \left.\frac{1}{\mathrm{a}+\mathrm{b} x} \int_{0}^{\eta} v(x, t) d t-\frac{1}{\mathrm{a}+\mathrm{b} x} \int_{0}^{\eta} e^{-\mathrm{b} t} v\left(\left(\frac{\mathrm{a}}{\mathrm{b}}+x\right) e^{-\mathrm{b}(\eta-t)}-\frac{\mathrm{a}}{\mathrm{b}(\eta-t)}, s\right) d t\right\} d s \\
& +\frac{\mathrm{b}}{\mathrm{a}+\mathrm{b} x} \int_{0}^{\eta} \int_{0}^{t} e^{\mathrm{b}(\eta-t)} v\left(\left(\frac{\mathrm{a}}{\mathrm{b}}+x\right) e^{-\mathrm{b}(\eta-t)}-\frac{\mathrm{a}}{\mathrm{b}}, s\right) d s d t .
\end{aligned}
$$

Therefore we know that (5.4) is equivalent to the following integral equation:

$$
\begin{aligned}
v(x, \eta)= & f\left(\left(\frac{\mathrm{a}}{\mathrm{b}}+x\right) e^{-\mathrm{b} \eta}-\frac{\mathrm{a}}{\mathrm{b}}, 0\right)+\int_{0}^{\eta} h\left(\left(\frac{\mathrm{a}}{\mathrm{b}}+x\right) e^{-\mathrm{b}(\eta-t)}-\frac{\mathrm{a}}{\mathrm{b}}, t\right) d t \\
& +\frac{\mathrm{c}}{\mathrm{a}+\mathrm{b} x} \int_{0}^{\eta} e^{\mathrm{b}(\eta-t)} v\left(\left(\frac{\mathrm{a}}{\mathrm{b}}+x\right) e^{-\mathrm{b}(\eta-t)}-\frac{\mathrm{a}}{\mathrm{b}}, t\right) d t \\
& -\frac{\mathrm{c}}{\mathrm{a}+\mathrm{b} x} \int_{0}^{\eta} v(x, t) d t \\
& -\frac{\mathrm{bc}}{\mathrm{a}+\mathrm{b} x} \int_{0}^{\eta} \int_{0}^{t} e^{\mathrm{b}(\eta-t)} v\left(\left(\frac{\mathrm{a}}{\mathrm{b}}+x\right) e^{-\mathrm{b}(\eta-t)}-\frac{\mathrm{a}}{\mathrm{b}}, s\right) d s d t .
\end{aligned}
$$

Let us prove that the solution $v(x, \eta)$ of $(5.5)$ satisfies the condition (BS), by using the iteration method. We define $\left\{v_{n}(x, \eta)\right\}_{n=0}^{\infty}$ as follows:

$$
v_{0}(x, \eta)=f\left(\left(\frac{\mathrm{a}}{\mathrm{b}}+x\right) e^{-\mathrm{b} \eta}-\frac{\mathrm{a}}{\mathrm{b}}, 0\right)+\int_{0}^{\eta} h\left(\left(\frac{\mathrm{a}}{\mathrm{b}}+x\right) e^{-\mathrm{b}(\eta-t)}-\frac{\mathrm{a}}{\mathrm{b}}, t\right) d t .
$$

For $n \geq 0$,

$$
\begin{aligned}
v_{n+1}(x, \eta)= & v_{0}(x, \eta)+\frac{\mathrm{c}}{\mathrm{a}+\mathrm{b} x} \int_{0}^{\eta} e^{\mathrm{b}(\eta-t)} v_{n}\left(\left(\frac{\mathrm{a}}{\mathrm{b}}+x\right) e^{-\mathrm{b}(\eta-t)}-\frac{\mathrm{a}}{\mathrm{b}}, t\right) d t \\
& -\frac{\mathrm{c}}{\mathrm{a}+\mathrm{b} x} \int_{0}^{\eta} v_{n}(x, t) d t
\end{aligned}
$$




$$
-\frac{\mathrm{bc}}{\mathrm{a}+\mathrm{b} x} \int_{0}^{\eta} \int_{0}^{t} e^{\mathrm{b}(\eta-t)} v_{n}\left(\left(\frac{\mathrm{a}}{\mathrm{b}}+x\right) e^{-\mathrm{b}(\eta-t)}-\frac{\mathrm{a}}{\mathrm{b}}, s\right) d s d t .
$$

Next, we put $w_{0}(x, \eta):=v_{0}(x, \eta)$ and $w_{n}(x, \eta)=v_{n}(x, \eta)-v_{n-1}(x, \eta)$ for $n \geq 1$, and we define $\widetilde{w}_{n}(x, \eta, t)$ by

$$
\widetilde{w}_{n}(x, \eta, t):=w_{n}\left(\left(\frac{\mathrm{a}}{\mathrm{b}}+x\right) e^{-\mathrm{b}(\eta-t)}-\frac{\mathrm{a}}{\mathrm{b}}, t\right) .
$$

Now let us take a monotonically decreasing positive sequence $\left\{\varepsilon_{n}\right\}_{n=0}^{\infty}$ satisfying (3.8). Furthermore let us take positive constants $K$ and $L$ so that

$$
\sup _{\eta \in E_{+}(\theta, \rho)}\left|e^{\mathrm{b} \eta}\right| \leq K, \sup _{|x| \leq r}\left|\frac{\mathrm{c}}{\mathrm{a}+\mathrm{b} x}\right| \leq L, \sup _{|x| \leq r}\left|\frac{\mathrm{bc}}{\mathrm{a}+\mathrm{b} x}\right| \leq L
$$

(by the assumption $\Re\left(-\mathrm{b} e^{i \theta}\right) \geq 0$ we can take such $\left.K\right)$. Then we obtain the following lemma:

Lemma 5.1. $\quad \widetilde{w}_{n}(x, \eta, t)$ is continued analytically to $\left\{(x, \eta, t) \in \mathbf{C}^{3} ;|x|\right.$ $\left.\leq r, \eta \in E_{+}\left(\theta, \rho-\sum_{j=0}^{n} \varepsilon_{j}\right), t \in G_{\eta}^{\varepsilon_{n}}\right\}$. Furthermore on $\left\{(x, \eta, t) \in \mathbf{C}^{3} ;|x| \leq\right.$ $\left.r, \eta \in E_{+}\left(\theta, \rho-\sum_{j=0}^{n} \varepsilon_{j}\right), t \in G_{\eta}\right\}$ we have the following estimate: For some positive constants $C_{1}$ and $\delta_{1}$,

$$
\left|\widetilde{w}_{n}\left(x, \eta, G_{\eta}(R)\right)\right| \leq C_{1} e^{\delta_{1}|\eta|}(L K)^{n} \sum_{k=0}^{n}\left(\begin{array}{l}
n \\
k
\end{array}\right) \frac{1}{\delta_{1}^{n-k}}\left(1+\frac{1}{\delta_{1}}\right)^{k} \frac{R^{k}}{k !}
$$

If we admit Lemma 5.1, the theorem is proved similarly to the previous cases.

Proof of Lemma 5.1. It is proved by the induction. We omit the proof for $n=0$ by the same reason as before. Let us assume that the lemma is proved up to $n$. Since $\left\{w_{n}(x, \eta)\right\}_{n=0}^{\infty}$ is determined by

$$
\begin{aligned}
w_{n+1}(x, \eta)= & \frac{\mathrm{c}}{\mathrm{a}+\mathrm{b} x} \int_{0}^{\eta} e^{\mathrm{b}(\eta-t)} w_{n}\left(\left(\frac{\mathrm{a}}{\mathrm{b}}+x\right) e^{-\mathrm{b}(\eta-t)}-\frac{\mathrm{a}}{\mathrm{b}}, t\right) d t \\
& -\frac{\mathrm{c}}{\mathrm{a}+\mathrm{b} x} \int_{0}^{\eta} w_{n}(x, t) d t \\
& -\frac{\mathrm{bc}}{\mathrm{a}+\mathrm{b} x} \int_{0}^{\eta} \int_{0}^{t} e^{\mathrm{b}(\eta-t)} w_{n}\left(\left(\frac{\mathrm{a}}{\mathrm{b}}+x\right) e^{-\mathrm{b}(\eta-t)}-\frac{\mathrm{a}}{\mathrm{b}}, s\right) d s d t
\end{aligned}
$$


we have

$$
\begin{aligned}
\widetilde{w}_{n+1}(x, \eta, t)= & w_{n+1}\left(\left(\frac{\mathrm{a}}{\mathrm{b}}+x\right) e^{-\mathrm{b}(\eta-t)}-\frac{\mathrm{a}}{\mathrm{b}}, t\right) \\
= & \frac{\mathrm{c}}{\mathrm{a}+\mathrm{b} x} \int_{0}^{t} e^{\mathrm{b}\left(\eta-s_{1}\right)} \widetilde{w}_{n}\left(x, \eta, s_{1}\right) d s_{1} \\
& -\frac{\mathrm{c}}{\mathrm{a}+\mathrm{b} x} \int_{0}^{t} e^{\mathrm{b}(\eta-t)} \widetilde{w}_{n}\left(x, \eta-t+s_{1}, s_{1}\right) d s_{1} \\
& -\frac{\mathrm{bc}}{\mathrm{a}+\mathrm{b} x} \int_{0}^{t} \int_{0}^{s_{1}} e^{\mathrm{b}\left(\eta-s_{1}\right)} \widetilde{w}_{n}\left(x, \eta-s_{1}+s_{2}, s_{2}\right) d s_{2} d s_{1} \\
= & I_{1}(x, \eta, t)-I_{2}(x, \eta, t)-I_{3}(x, \eta, t) .
\end{aligned}
$$

Let us prove that each $I_{i}(x, \eta, t)$ is well-defined on $\left\{(x, \eta, t) \in \mathbf{C}^{3} ;|x| \leq\right.$ $\left.r, \eta \in E_{+}\left(\theta, \rho-\sum_{j=0}^{n+1} \varepsilon_{j}\right), t \in G_{\eta}^{\varepsilon_{n+1}}\right\}$. We put $t=G_{\eta}(\tau)\left(\tau \in U_{\varepsilon_{n+1}}[0,|\eta|]\right)$.

On $I_{1}\left(x, \eta, G_{\eta}(\tau)\right)$ : It is clear that $\eta \in E_{+}\left(\theta, \rho-\sum_{j=0}^{n+1} \varepsilon_{j}\right) \subset E_{+}(\theta, \rho-$ $\left.\sum_{j=0}^{n} \varepsilon_{j}\right)$. By taking an integral path as $s_{1}\left(\sigma_{1}\right)=G_{\eta}\left(\sigma_{1}\right)\left(\sigma_{1} \in[0, \tau]\right)$, it holds that $s_{1}\left(\sigma_{1}\right) \in G_{\eta}^{\varepsilon_{n+1}} \subset G_{\eta}^{\varepsilon_{n}}$. Hence $\widetilde{w}_{n}\left(x, \eta, s_{1}\left(\sigma_{1}\right)\right)$ is well-defined and $I_{1}\left(x, \eta, G_{\eta}(\tau)\right)$ is well-defined.

On $I_{2}\left(x, \eta, G_{\eta}(\tau)\right)$ : By taking an integral path as $s_{1}\left(\sigma_{1}\right)=G_{\eta}\left(\sigma_{1}\right)\left(\sigma_{1} \in\right.$ $[0, \tau])$, it holds that $\eta-G_{\eta}(\tau)+s_{1}\left(\sigma_{1}\right) \in E_{+}\left(\theta, \rho-\sum_{j=0}^{n} \varepsilon_{j}\right)$ and $s_{1}\left(\sigma_{1}\right) \in$ $G_{\eta-G_{\eta}(\tau)+s_{1}\left(\sigma_{1}\right)}^{\varepsilon_{n+1}} \subset G_{\eta-G_{\eta}(\tau)+s_{1}\left(\sigma_{1}\right)}^{\varepsilon_{n}}$. Hence $\widetilde{w}_{n}\left(x, \eta-G_{\eta}(\tau)+s_{1}\left(\sigma_{1}\right), s_{1}\left(\sigma_{1}\right)\right)$ is well-defined and $I_{2}\left(x, \eta, G_{\eta}(\tau)\right)$ is well-defined.

On $I_{3}\left(x, \eta, G_{\eta}(\tau)\right)$ : By taking integral paths as $s_{1}\left(\sigma_{1}\right)=G_{\eta}\left(\sigma_{1}\right)\left(\sigma_{1} \in\right.$ $[0, \tau])$ and $s_{2}\left(\sigma_{2}\right)=G_{\eta}\left(\sigma_{2}\right)\left(\sigma_{2} \in\left[0, \sigma_{1}\right]\right)$, it holds that $\eta-s_{1}\left(\sigma_{1}\right)+s_{2}\left(\sigma_{2}\right) \in$ $E_{+}\left(\theta, \rho-\sum_{j=0}^{n} \varepsilon_{j}\right)$ and $s_{2}\left(\sigma_{2}\right) \in G_{\eta-s_{1}\left(\sigma_{1}\right)+s_{2}\left(\sigma_{2}\right)}^{\varepsilon_{n+1}} \subset G_{\eta-s_{1}\left(\sigma_{1}\right)+s_{2}\left(\sigma_{2}\right)}^{\varepsilon_{n}}$. Hence $\widetilde{w}_{n}\left(x, \eta-s_{1}\left(\sigma_{1}\right)+s_{2}\left(\sigma_{2}\right), s_{2}\left(\sigma_{2}\right)\right)$ is well-defined and $I_{3}\left(x, \eta, G_{\eta}(\tau)\right)$ is welldefined.

Therefore $\widetilde{w}_{n+1}(x, \eta, t)$ is well-defined and holomorphic on $\{(x, \eta, t) \in$ $\left.\mathbf{C}^{3} ;|x| \leq r, \eta \in E_{+}\left(\theta, \rho-\sum_{j=0}^{n+1} \varepsilon_{j}\right), t \in G_{\eta}^{\varepsilon_{n+1}}\right\}$. Moreover on $\{(x, \eta, t) \in$ $\left.\mathbf{C}^{3} ;|x| \leq r, \eta \in E_{+}\left(\theta, \rho-\sum_{j=0}^{n+1} \varepsilon_{j}\right), t \in G_{\eta}\right\}$ we have the following representations:

$$
\begin{aligned}
& I_{1}\left(x, \eta, G_{\eta}(R)\right) \\
& =\frac{\mathrm{c}}{\mathrm{a}+\mathrm{b} x} \int_{0}^{R} \exp \left[\mathrm{b}\left(|\eta|-R_{1}\right) e^{i \arg (\eta)}\right] \widetilde{w}_{n}\left(x, \eta, G_{\eta}\left(R_{1}\right)\right) e^{i \arg (\eta)} d R_{1}, \\
& I_{2}\left(x, \eta, G_{\eta}(R)\right) \\
& =\frac{\mathrm{c}}{\mathrm{a}+\mathrm{b} x} \int_{0}^{R} \exp \left[\mathrm{b}(|\eta|-R) e^{i \arg (\eta)}\right] \\
& \quad \times \widetilde{w}_{n}\left(x,\left(|\eta|-R+R_{1}\right) e^{i \arg (\eta)}, G_{\left.\left(|\eta|-R+R_{1}\right) e^{i \arg (\eta)}\left(R_{1}\right)\right) e^{i \arg (\eta)} d R_{1},}\right.
\end{aligned}
$$




$$
\begin{aligned}
& I_{3}\left(x, \eta, G_{\eta}(R)\right) \\
& =\frac{\mathrm{bc}}{\mathrm{a}+\mathrm{b} x} \int_{0}^{R} \int_{0}^{R_{1}} \exp \left[\mathrm{b}\left(|\eta|-R_{1}\right) e^{i \arg (\eta)}\right] \\
& \times \widetilde{w}_{n}\left(x,\left(|\eta|-R_{1}+R_{2}\right) e^{i \arg (\eta)},\right. \\
& \quad G_{\left.\left(|\eta|-R_{1}+R_{2}\right) e^{i \arg (\eta)}\left(R_{2}\right)\right)\left\{e^{i \arg (\eta)}\right\}^{2} d R_{2} d R_{1} .}
\end{aligned}
$$

Let us estimate each $I_{i}\left(x, \eta, G_{\eta}(R)\right)$.

On $I_{1}\left(x, \eta, G_{\eta}(R)\right)$ : It follows from the assumption of the induction that

$$
\left|\widetilde{w}_{n}\left(x, \eta, G_{\eta}\left(R_{1}\right)\right)\right| \leq C_{1} e^{\delta_{1}|\eta|}(L K)^{n} \sum_{k=0}^{n}\left(\begin{array}{l}
n \\
k
\end{array}\right) \frac{1}{\delta_{1}^{n-k}}\left(1+\frac{1}{\delta_{1}}\right)^{k} \frac{R_{1}^{k}}{k !},
$$

which implies that

$$
\begin{aligned}
\left|I_{1}\left(x, \eta, G_{\eta}(R)\right)\right| & \leq C_{1} e^{\delta_{1}|\eta|}(L K)^{n+1} \sum_{k=0}^{n}\left(\begin{array}{l}
n \\
k
\end{array}\right) \frac{1}{\delta_{1}^{n-k}}\left(1+\frac{1}{\delta_{1}}\right)^{k} \int_{0}^{R} \frac{R_{1}^{k}}{k !} d R_{1} \\
& =C_{1} e^{\delta_{1}|\eta|}(L K)^{n+1} \sum_{k=0}^{n}\left(\begin{array}{l}
n \\
k
\end{array}\right) \frac{1}{\delta_{1}^{n-k}}\left(1+\frac{1}{\delta_{1}}\right)^{k} \frac{R^{k+1}}{(k+1) !} .
\end{aligned}
$$

On $I_{2}\left(x, \eta, G_{\eta}(R)\right)$ : By the assumption of the induction, we have

$$
\begin{aligned}
& \mid \widetilde{w}_{n}\left(x,\left(|\eta|-R+R_{1}\right) e^{i \arg (\eta)}, G_{\left.\left(|\eta|-R+R_{1}\right) e^{i \arg (\eta)}\left(R_{1}\right)\right) \mid}\right. \\
& \quad \leq C_{1} e^{\delta_{1}|\eta|} e^{-\delta_{1} R} e^{\delta_{1} R_{1}}(L K)^{n} \sum_{k=0}^{n}\left(\begin{array}{l}
n \\
k
\end{array}\right) \frac{1}{\delta_{1}^{n-k}}\left(1+\frac{1}{\delta_{1}}\right)^{k} \frac{R_{1}^{k}}{k !}
\end{aligned}
$$

which implies that

$$
\begin{aligned}
& \left|I_{2}\left(x, \eta, G_{\eta}(R)\right)\right| \\
& \quad \leq C_{1} e^{\delta_{1}|\eta|}(L K)^{n+1} \sum_{k=0}^{n}\left(\begin{array}{l}
n \\
k
\end{array}\right) \frac{1}{\delta_{1}^{n-k}}\left(1+\frac{1}{\delta_{1}}\right)^{k} e^{-\delta_{1} R} \int_{0}^{R} e^{\delta_{1} R_{1}} \frac{R_{1}^{k}}{k !} d R_{1} \\
& \quad \leq C_{1} e^{\delta_{1}|\eta|}(L K)^{n+1} \sum_{k=0}^{n}\left(\begin{array}{l}
n \\
k
\end{array}\right) \frac{1}{\delta_{1}^{n+1-k}}\left(1+\frac{1}{\delta_{1}}\right)^{k} \frac{R^{k}}{k !}
\end{aligned}
$$

(see $(3.11))$.

On $I_{3}\left(x, \eta, G_{\eta}(R)\right)$ : The assumption of the induction implies that

$$
\begin{aligned}
& \mid \widetilde{w}_{n}\left(x,\left(|\eta|-R_{1}+R_{2}\right) e^{i \arg (\eta)}, G_{\left.\left(|\eta|-R_{1}+R_{2}\right) e^{i \arg (\eta)}\left(R_{2}\right)\right) \mid}\right. \\
& \quad \leq C_{1} e^{\delta_{1}|\eta|} e^{-\delta_{1} R_{1}} e^{\delta_{1} R_{2}}(L K)^{n} \sum_{k=0}^{n}\left(\begin{array}{l}
n \\
k
\end{array}\right) \frac{1}{\delta_{1}^{n-k}}\left(1+\frac{1}{\delta_{1}}\right)^{k} \frac{R_{2}^{k}}{k !} .
\end{aligned}
$$


Hence we obtain that

$$
\begin{aligned}
& \left|I_{3}\left(x, \eta, G_{\eta}(R)\right)\right| \\
& \leq C_{1} e^{\delta_{1}|\eta|}(L K)^{n+1} \sum_{k=0}^{n}\left(\begin{array}{l}
n \\
k
\end{array}\right) \frac{1}{\delta_{1}^{n-k}}\left(1+\frac{1}{\delta_{1}}\right)^{k} \\
& \quad \times \int_{0}^{R} e^{-\delta_{1} R_{1}} \int_{0}^{R_{1}} e^{\delta_{1} R_{2}} \frac{R_{2}^{k}}{k !} d R_{2} d R_{1} \\
& \leq C_{1} e^{\delta_{1}|\eta|}(L K)^{n+1} \sum_{k=0}^{n}\left(\begin{array}{l}
n \\
k
\end{array}\right) \frac{1}{\delta_{1}^{n+1-k}}\left(1+\frac{1}{\delta_{1}}\right)^{k} \int_{0}^{R} \frac{R_{1}^{k}}{k !} d R_{1} \text { (see (3.11)) } \\
& =C_{1} e^{\delta_{1}|\eta|}(L K)^{n+1} \sum_{k=0}^{n}\left(\begin{array}{l}
n \\
k
\end{array}\right) \frac{1}{\delta_{1}^{n+1-k}}\left(1+\frac{1}{\delta_{1}}\right)^{k} \frac{R^{k+1}}{(k+1) !} .
\end{aligned}
$$

Therefore we have

$$
\begin{aligned}
\left|\widetilde{w}_{n+1}\left(x, \eta, G_{\eta}(R)\right)\right| & \leq C_{1} e^{\delta_{1}|\eta|}(L K)^{n+1} \times\left\{\sum_{k=0}^{n}\left(\begin{array}{l}
n \\
k
\end{array}\right) \frac{1}{\delta_{1}^{n-k}}\left(1+\frac{1}{\delta_{1}}\right)^{k} \frac{R^{k+1}}{(k+1) !}\right. \\
& \left.+\sum_{k=0}^{n}\left(\begin{array}{l}
n \\
k
\end{array}\right) \frac{1}{\delta_{1}^{n+1-k}}\left(1+\frac{1}{\delta_{1}}\right)^{k} \frac{R^{k}}{k !}+\sum_{k=0}^{n}\left(\begin{array}{l}
n \\
k
\end{array}\right) \frac{1}{\delta_{1}^{n+1-k}}\left(1+\frac{1}{\delta_{1}}\right)^{k} \frac{R^{k+1}}{(k+1) !}\right\} \\
= & C_{1} e^{\delta_{1}|\eta|}(L K)^{n+1} \times\left\{\sum_{k=1}^{n+1}\left(\begin{array}{c}
n \\
k-1
\end{array}\right) \frac{1}{\delta_{1}^{n+1-k}}\left(1+\frac{1}{\delta_{1}}\right)^{k-1} \frac{R^{k}}{k !}\right. \\
& \left.+\sum_{k=0}^{n}\left(\begin{array}{l}
n \\
k
\end{array}\right) \frac{1}{\delta_{1}^{n+1-k}}\left(1+\frac{1}{\delta_{1}}\right)^{k} \frac{R^{k}}{k !}+\sum_{k=1}^{n+1}\left(\begin{array}{c}
n \\
k-1
\end{array}\right) \frac{1}{\delta_{1}^{n+2-k}}\left(1+\frac{1}{\delta_{1}}\right)^{k-1} \frac{R^{k}}{k !}\right\} \\
= & C_{1} e^{\delta_{1}|\eta|}(L K)^{n+1} \times\left\{\frac{1}{\delta_{1}^{n+1}}+\sum_{k=1}^{n} A\left(n, k, \delta_{1}\right) \frac{R^{k}}{k !}+\left(1+\frac{1}{\delta_{1}}\right)^{n} \frac{R^{n+1}}{(n+1) !}\right. \\
& \left.+\frac{1}{\delta_{1}}\left(1+\frac{1}{\delta_{1}}\right)^{n} \frac{R^{n+1}}{(n+1) !}\right\},
\end{aligned}
$$

where

$$
\begin{aligned}
A\left(n, k, \delta_{1}\right)= & \left(\begin{array}{c}
n \\
k-1
\end{array}\right) \frac{1}{\delta_{1}^{n+1-k}}\left(1+\frac{1}{\delta_{1}}\right)^{k-1}+\left(\begin{array}{l}
n \\
k
\end{array}\right) \frac{1}{\delta_{1}^{n+1-k}}\left(1+\frac{1}{\delta_{1}}\right)^{k} \\
& +\left(\begin{array}{c}
n \\
k-1
\end{array}\right) \frac{1}{\delta_{1}^{n+2-k}}\left(1+\frac{1}{\delta_{1}}\right)^{k-1} .
\end{aligned}
$$


Here it holds that

$$
\begin{aligned}
A\left(n, k, \delta_{1}\right) & =\frac{1}{\delta_{1}^{n+1-k}}\left(1+\frac{1}{\delta_{1}}\right)^{k-1}\left\{\left(\begin{array}{c}
n \\
k-1
\end{array}\right)+\left(\begin{array}{l}
n \\
k
\end{array}\right)\left(1+\frac{1}{\delta_{1}}\right)+\left(\begin{array}{c}
n \\
k-1
\end{array}\right) \frac{1}{\delta_{1}}\right\} \\
& =\frac{1}{\delta_{1}^{n+1-k}}\left(1+\frac{1}{\delta_{1}}\right)^{k}\left\{\left(\begin{array}{l}
n \\
k
\end{array}\right)+\left(\begin{array}{c}
n \\
k-1
\end{array}\right)\right\} \\
& =\left(\begin{array}{c}
n+1 \\
k
\end{array}\right) \frac{1}{\delta_{1}^{n+1-k}}\left(1+\frac{1}{\delta_{1}}\right)^{k}
\end{aligned}
$$

and

$$
\left(1+\frac{1}{\delta_{1}}\right)^{n} \frac{R^{n+1}}{(n+1) !}+\frac{1}{\delta_{1}}\left(1+\frac{1}{\delta_{1}}\right)^{n} \frac{R^{n+1}}{(n+1) !}=\left(1+\frac{1}{\delta_{1}}\right)^{n+1} \frac{R^{n+1}}{(n+1) !} .
$$

Therefore we obtain

$$
\left|\widetilde{w}_{n+1}\left(x, \eta, G_{\eta}(R)\right)\right| \leq C_{1} e^{\delta_{1}|\eta|}(L K)^{n+1} \sum_{k=0}^{n+1}\left(\begin{array}{c}
n+1 \\
k
\end{array}\right) \frac{1}{\delta_{1}^{n+1-k}}\left(1+\frac{1}{\delta_{1}}\right)^{k} \frac{R^{k}}{k !},
$$

which implies the lemma for $n+1$. The proof is completed.

\section{§6. The Proof of Theorem 1.2 (Case (4))}

Let us prove that the solution $v(x, \eta)$ of $(2.5)$ satisfies the condition (BS) in Case $(4)(\mathrm{b}, \mathrm{d} \neq 0)$. First, we remark that in general the solution $w(x, \eta)$ of the initial value problem of the following first order linear partial differential equation

$$
\begin{gathered}
\left\{(1+\mathrm{d} \eta) D_{\eta}+(\mathrm{a}+\mathrm{b} x) D_{x}\right\} w(x, \eta)=k(x, \eta), \\
w(x, 0)=l(x)
\end{gathered}
$$

is given by

$$
\begin{aligned}
w(x, \eta)= & l\left(\left(\frac{\mathrm{a}}{\mathrm{b}}+x\right)(1+\mathrm{d} \eta)^{-\mathrm{b} / \mathrm{d}}-\frac{\mathrm{a}}{\mathrm{b}}\right) \\
& +\int_{0}^{\eta} k\left(\left(\frac{\mathrm{a}}{\mathrm{b}}+x\right)\left(\frac{1+\mathrm{d} \eta}{1+\mathrm{d} t}\right)^{-\mathrm{b} / \mathrm{d}}-\frac{\mathrm{a}}{\mathrm{b}}, t\right) \frac{1}{1+\mathrm{d} t} d t .
\end{aligned}
$$

Proof of the theorem. Similarly to the previous cases, we assume that $\mathrm{c} \neq 0$. Therefore we remark that $\Re(-\mathrm{b} / \mathrm{d})>-1$ by the assumption (1.13). The equation (2.5) is rewritten as follows:

$$
\begin{gathered}
\left\{(1+\mathrm{d} \eta) D_{\eta}+(\mathrm{a}+\mathrm{b} x) D_{x}\right\} v(x, \eta)=-\mathrm{c} \int_{0}^{\eta} v_{x}(x, s) d s+h(x, \eta), \\
v(x, 0)=f(x, 0) .
\end{gathered}
$$


Furthermore we can see that (6.3) is equivalent to the following integral equation in a similar way to the previous cases:

$$
\begin{aligned}
v(x, \eta)= & f\left(\left(\frac{\mathrm{a}}{\mathrm{b}}+x\right)(1+\mathrm{d} \eta)^{-\mathrm{b} / \mathrm{d}}-\frac{\mathrm{a}}{\mathrm{b}}, 0\right) \\
& +\int_{0}^{\eta} h\left(\left(\frac{\mathrm{a}}{\mathrm{b}}+x\right)\left(\frac{1+\mathrm{d} \eta}{1+\mathrm{d} t}\right)^{-\mathrm{b} / \mathrm{d}}-\frac{\mathrm{a}}{\mathrm{b}}, t\right) \frac{1}{1+\mathrm{d} t} d t \\
& +\frac{\mathrm{c}}{\mathrm{b}}\left(\frac{\mathrm{a}}{\mathrm{b}}+x\right)^{-1}(1+\mathrm{d} \eta)^{\mathrm{b} / \mathrm{d}} \\
& \times \int_{0}^{\eta}(1+\mathrm{d} t)^{-\mathrm{b} / \mathrm{d}} v\left(\left(\frac{\mathrm{a}}{\mathrm{b}}+x\right)\left(\frac{1+\mathrm{d} \eta}{1+\mathrm{d} t}\right)^{-\mathrm{b} / \mathrm{d}}-\frac{\mathrm{a}}{\mathrm{b}}, t\right) d t \\
& -\frac{\mathrm{c}}{\mathrm{b}}\left(\frac{\mathrm{a}}{\mathrm{b}}+x\right)^{-1} \int_{0}^{\eta} v(x, t) d t \\
& -\mathrm{c}\left(\frac{\mathrm{a}}{\mathrm{b}}+x\right)^{-1}(1+\mathrm{d} \eta)^{\mathrm{b} / \mathrm{d}} \int_{0}^{\eta} \int_{0}^{t}(1+\mathrm{d} t)^{-\mathrm{b} / \mathrm{d}} \\
& \times v\left(\left(\frac{\mathrm{a}}{\mathrm{b}}+x\right)\left(\frac{1+\mathrm{d} \eta}{1+\mathrm{d} t}\right)^{-\mathrm{b} / \mathrm{d}}-\frac{\mathrm{a}}{\mathrm{b}}, s\right) \frac{1}{1+\mathrm{d} t} d s d t .
\end{aligned}
$$

We prove that the solution $v(x, \eta)$ of $(6.4)$ satisfies the condition (BS) by using the iteration method. Let us define $\left\{v_{n}(x, \eta)\right\}_{n=0}^{\infty}$ as follows:

$$
\begin{aligned}
v_{0}(x, \eta)= & f\left(\left(\frac{\mathrm{a}}{\mathrm{b}}+x\right)(1+\mathrm{d} \eta)^{-\mathrm{b} / \mathrm{d}}-\frac{\mathrm{a}}{\mathrm{b}}, 0\right) \\
& +\int_{0}^{\eta} h\left(\left(\frac{\mathrm{a}}{\mathrm{b}}+x\right)\left(\frac{1+\mathrm{d} \eta}{1+\mathrm{d} t}\right)^{-\mathrm{b} / \mathrm{d}}-\frac{\mathrm{a}}{\mathrm{b}}, t\right) \frac{1}{1+\mathrm{d} t} d t .
\end{aligned}
$$

For $n \geq 0$,

$$
\begin{aligned}
v_{n+1}(x, \eta)= & v_{0}(x, \eta) \\
+ & \frac{\mathrm{c}}{\mathrm{b}}\left(\frac{\mathrm{a}}{\mathrm{b}}+x\right)^{-1}(1+\mathrm{d} \eta)^{\mathrm{b} / \mathrm{d}} \\
& \quad \times \int_{0}^{\eta}(1+\mathrm{d} t)^{-\mathrm{b} / \mathrm{d}} v_{n}\left(\left(\frac{\mathrm{a}}{\mathrm{b}}+x\right)\left(\frac{1+\mathrm{d} \eta}{1+\mathrm{d} t}\right)^{-\mathrm{b} / \mathrm{d}}-\frac{\mathrm{a}}{\mathrm{b}}, t\right) d t \\
& -\frac{\mathrm{c}}{\mathrm{b}}\left(\frac{\mathrm{a}}{\mathrm{b}}+x\right)^{-1} \int_{0}^{\eta} v_{n}(x, t) d t \\
& -\mathrm{c}\left(\frac{\mathrm{a}}{\mathrm{b}}+x\right)^{-1}(1+\mathrm{d} \eta)^{\mathrm{b} / \mathrm{d}} \int_{0}^{\eta} \int_{0}^{t}(1+\mathrm{d} t)^{-\mathrm{b} / \mathrm{d}}
\end{aligned}
$$




$$
\times v_{n}\left(\left(\frac{\mathrm{a}}{\mathrm{b}}+x\right)\left(\frac{1+\mathrm{d} \eta}{1+\mathrm{d} t}\right)^{-\mathrm{b} / \mathrm{d}}-\frac{\mathrm{a}}{\mathrm{b}}, s\right) \frac{1}{1+\mathrm{d} t} d s d t .
$$

Next we put $w_{0}(x, \eta):=v_{0}(x, \eta)$ and $w_{n}(x, \eta):=v_{n}(x, \eta)-v_{n-1}(x, \eta)$ for $n \geq 1$, and we define $\widetilde{w}_{n}(x, \eta, t)$ by

$$
\widetilde{w}_{n}(x, \eta, t):=w_{n}\left(\left(\frac{\mathrm{a}}{\mathrm{b}}+x\right)\left(\frac{1+\mathrm{d} \eta}{1+\mathrm{d} t}\right)^{-\mathrm{b} / \mathrm{d}}-\frac{\mathrm{a}}{\mathrm{b}}, t\right) .
$$

Now let us define $0 \leq p<1$ by

$$
p= \begin{cases}0 & (\text { if } \Re(-\mathrm{b} / \mathrm{d}) \geq 0) \\ \Re(\mathrm{b} / \mathrm{d}) & (\text { if } 0>\Re(-\mathrm{b} / \mathrm{d})>-1),\end{cases}
$$

and let us take a monotonically decreasing positive sequence $\left\{\varepsilon_{n}\right\}_{n=0}^{\infty}$ satisfying (3.8). Furthermore we take $K>1$ so that (4.8) holds for $\eta \in E_{+}(\theta, \rho)$, and we take positive constants $L$ and $M$ so that

$$
\left|(1+\mathrm{d} \eta)^{\mathrm{b} / \mathrm{d}}\right| \leq L(1+|\eta|)^{p}
$$

for $\eta \in E_{+}(\theta, \rho)$ and

$$
\sup _{|x| \leq r}\left|\frac{\mathrm{c}}{\mathrm{b}}\left(\frac{\mathrm{a}}{\mathrm{b}}+x\right)^{-1}\right| \leq M, \sup _{|x| \leq r}\left|\mathrm{c}\left(\frac{\mathrm{a}}{\mathrm{b}}+x\right)^{-1}\right| \leq M .
$$

Then we obtain the following lemma:

Lemma 6.1. $\widetilde{w}_{n}(x, \eta, t)$ is continued analytically to $\left\{(x, \eta, t) \in \mathbf{C}^{3} ;|x|\right.$ $\left.\leq r, \eta \in E_{+}\left(\theta, \rho-\sum_{j=0}^{n} \varepsilon_{j}\right), t \in \widetilde{G}_{\eta}^{\varepsilon_{n}}\right\}$. Furthermore on $\left\{(x, \eta, t) \in \mathbf{C}^{3} ;|x| \leq\right.$ $\left.r, \eta \in E_{+}\left(\theta, \rho-\sum_{j=0}^{n} \varepsilon_{j}\right), t \in \widetilde{G}_{\eta}\right\}$ we have the following estimate: For some positive constants $C_{1}$ and $\delta_{1}$,

$$
\begin{aligned}
\left|\widetilde{w}_{n}\left(x, \eta, \widetilde{G}_{\eta}(R)\right)\right| \leq & C_{1} e^{\delta_{1}|\eta|}\left(M L K^{3}\right)^{n} \frac{1}{(1+|\eta|-R)^{n(1-p)}} \\
& \times \sum_{k=0}^{n}\left(\begin{array}{l}
n \\
k
\end{array}\right)\left\{\frac{1}{\delta_{1}}\left(1+\frac{1}{1-p}\right)\right\}^{n-k} \frac{(1+|\eta|)^{k}}{k !(1-p)^{k}} .
\end{aligned}
$$

If we admit Lemma 6.1 , the theorem is proved similarly to the previous cases.

Proof of Lemma 6.1. It is proved by the induction. We omit the proof for $n=0$. Let us assume that the lemma is proved up to $n$. Since $\left\{w_{n}(x, \eta)\right\}_{n=0}^{\infty}$ 
is determined by

$$
\begin{aligned}
w_{n+1}(x, \eta)= & \frac{\mathrm{c}}{\mathrm{b}}\left(\frac{\mathrm{a}}{\mathrm{b}}+x\right)^{-1}(1+\mathrm{d} \eta)^{\mathrm{b} / \mathrm{d}} \\
& \times \int_{0}^{\eta}(1+\mathrm{d} t)^{-\mathrm{b} / \mathrm{d}} w_{n}\left(\left(\frac{\mathrm{a}}{\mathrm{b}}+x\right)\left(\frac{1+\mathrm{d} \eta}{1+\mathrm{d} t}\right)^{-\mathrm{b} / \mathrm{d}}-\frac{\mathrm{a}}{\mathrm{b}}, t\right) d t \\
- & \frac{\mathrm{c}}{\mathrm{b}}\left(\frac{\mathrm{a}}{\mathrm{b}}+x\right)^{-1} \int_{0}^{\eta} w_{n}(x, t) d t \\
- & \mathrm{c}\left(\frac{\mathrm{a}}{\mathrm{b}}+x\right)^{-1}(1+\mathrm{d} \eta)^{\mathrm{b} / \mathrm{d}} \int_{0}^{\eta} \int_{0}^{t}(1+\mathrm{d} t)^{-\mathrm{b} / \mathrm{d}} \\
& \times w_{n}\left(\left(\frac{\mathrm{a}}{\mathrm{b}}+x\right)\left(\frac{1+\mathrm{d} \eta}{1+\mathrm{d} t}\right)^{-\mathrm{b} / \mathrm{d}}-\frac{\mathrm{a}}{\mathrm{b}}, s\right) \frac{1}{1+\mathrm{d} t} d s d t,
\end{aligned}
$$

we have

$$
\begin{aligned}
& \widetilde{w}_{n+1}(x, \eta, t) \\
&=w_{n+1}\left(\left(\frac{\mathrm{a}}{\mathrm{b}}+x\right)\left(\frac{1+\mathrm{d} \eta}{1+\mathrm{d} t}\right)^{-\mathrm{b} / \mathrm{d}}-\frac{\mathrm{a}}{\mathrm{b}}, t\right) \\
&=\frac{\mathrm{c}}{\mathrm{b}}\left(\frac{\mathrm{a}}{\mathrm{b}}+x\right)^{-1}(1+\mathrm{d} \eta)^{\mathrm{b} / \mathrm{d}} \int_{0}^{t}\left(1+\mathrm{d} s_{1}\right)^{-\mathrm{b} / \mathrm{d}} \widetilde{w}_{n}\left(x, \eta, s_{1}\right) d s_{1} \\
& \quad-\frac{\mathrm{c}}{\mathrm{b}}\left(\frac{\mathrm{a}}{\mathrm{b}}+x\right)^{-1}(1+\mathrm{d} \eta)^{\mathrm{b} / \mathrm{d}} \int_{0}^{t}(1+\mathrm{d} t)^{-\mathrm{b} / \mathrm{d}} \widetilde{w}_{n}\left(x, \zeta\left(\eta, t, s_{1}\right), s_{1}\right) d s_{1} \\
&-\mathrm{c}\left(\frac{\mathrm{a}}{\mathrm{b}}+x\right)^{-1}(1+\mathrm{d} \eta)^{\mathrm{b} / \mathrm{d}} \int_{0}^{t} \int_{0}^{s_{1}}\left(1+\mathrm{d} s_{1}\right)^{-\mathrm{b} / \mathrm{d}} \widetilde{w}_{n}\left(x, \varphi\left(\eta, s_{1}, s_{2}\right), s_{2}\right) \\
& \times \frac{1}{1+\mathrm{d} s_{1}} d s_{2} d s_{1} \\
&=: I_{1}(x, \eta, t)-I_{2}(x, \eta, t)-I_{3}(x, \eta, t),
\end{aligned}
$$

where $\zeta\left(\eta, t, s_{1}\right)$ and $\varphi\left(\eta, s_{1}, s_{2}\right)$ are defined by

$$
\frac{(1+\mathrm{d} \eta)\left(1+\mathrm{d} s_{1}\right)}{1+\mathrm{d} t}=1+\mathrm{d} \zeta\left(\eta, t, s_{1}\right)
$$

and

$$
\frac{(1+\mathrm{d} \eta)\left(1+\mathrm{d} s_{2}\right)}{1+\mathrm{d} s_{1}}=1+\mathrm{d} \varphi\left(\eta, s_{1}, s_{1}\right) .
$$


Let us prove that each $I_{i}(x, \eta, t)$ is well-defined on $\left\{(x, \eta, t) \in \mathbf{C}^{3} ;|x| \leq\right.$ $\left.r, \eta \in E_{+}\left(\theta, \rho-\sum_{j=0}^{n+1} \varepsilon_{j}\right), t \in \widetilde{G}_{\eta}^{\varepsilon_{n+1}}\right\}$. We put $t=\widetilde{G}_{\eta}(\tau)\left(\tau \in U_{\varepsilon_{n+1}}[0,|\eta|]\right)$.

On $I_{1}\left(x, \eta, \widetilde{G}_{\eta}(\tau)\right)$ : It is clear that $\eta \in E_{+}\left(\theta, \rho-\sum_{j=0}^{n+1} \varepsilon_{j}\right) \subset E_{+}(\theta, \rho-$ $\left.\sum_{j=0}^{n} \varepsilon_{j}\right)$. By taking an integral path as

$$
s_{1}\left(\sigma_{1}\right)=\widetilde{G}_{\eta}\left(\sigma_{1}\right)=\frac{\sigma_{1} e^{i \arg (\eta)}}{1+\mathrm{d}\left(|\eta|-\sigma_{1}\right) e^{i \arg (\eta)}}\left(\sigma_{1} \in[0, \tau]\right),
$$

it holds that $s_{1}\left(\sigma_{1}\right) \in \widetilde{G}_{\eta}^{\varepsilon_{n+1}} \subset \widetilde{G}_{\eta}^{\varepsilon_{n}}$. Hence $\widetilde{w}_{n}\left(x, \eta, s_{1}\left(\sigma_{1}\right)\right)$ is well-defined and we have the well-definedness for $I_{1}\left(x, \eta, \widetilde{G}_{\eta}(\tau)\right)$.

On $I_{2}\left(x, \eta, \widetilde{G}_{\eta}(\tau)\right)$ : By taking an integral path as

$$
s_{1}\left(\sigma_{1}\right)=\frac{\sigma_{1} e^{i \arg (\eta)}}{1+\mathrm{d}(|\eta|-\tau) e^{i \arg (\eta)}}\left(\sigma_{1} \in[0, \tau]\right),
$$

we have

$$
\begin{aligned}
\zeta\left(\eta, \widetilde{G}_{\eta}(\tau), s_{1}\left(\sigma_{1}\right)\right) & \in E_{+}\left(\theta, \rho-\sum_{j=0}^{n} \varepsilon_{j}\right) \text { and } \\
s_{1}\left(\sigma_{1}\right) & \in \widetilde{G}_{\zeta\left(\eta, \widetilde{G}_{\eta}(\tau), s_{1}\left(\sigma_{1}\right)\right)}^{\varepsilon_{n+1}} \subset \widetilde{G}_{\zeta\left(\eta, \widetilde{G}_{\eta}(\tau), s_{1}\left(\sigma_{1}\right)\right)}^{\varepsilon_{n}} .
\end{aligned}
$$

Hence $\widetilde{w}_{n}\left(x, \zeta\left(\eta, \widetilde{G}_{\eta}(\tau), s_{1}\left(\sigma_{1}\right)\right), s_{1}\left(\sigma_{1}\right)\right)$ is well-defined and $I_{2}\left(x, \eta, \widetilde{G}_{\eta}(\tau)\right)$ is well-defined.

On $I_{3}\left(x, \eta, \widetilde{G}_{\eta}(\tau)\right)$ : By taking integral paths as

$$
s_{1}\left(\sigma_{1}\right)=\widetilde{G}_{\eta}\left(\sigma_{1}\right)=\frac{\sigma_{1} e^{i \arg (\eta)}}{1+\mathrm{d}\left(|\eta|-\sigma_{1}\right) e^{i \arg (\eta)}}\left(\sigma_{1} \in[0, \tau]\right)
$$

and

$$
s_{2}\left(\sigma_{2}\right)=\frac{\sigma_{2} e^{i \arg (\eta)}}{1+\mathrm{d}\left(|\eta|-\sigma_{1}\right) e^{i \arg (\eta)}}\left(\sigma_{2} \in\left[0, \sigma_{1}\right]\right)
$$

it holds that

$$
\begin{aligned}
\varphi\left(\eta, s_{1}\left(\sigma_{1}\right), s_{2}\left(\sigma_{2}\right)\right) & \in E_{+}\left(\theta, \rho-\sum_{j=0}^{n} \varepsilon_{j}\right) \text { and } \\
s_{2}\left(\sigma_{2}\right) & \in \widetilde{G}_{\varphi\left(\eta, s_{1}\left(\sigma_{1}\right), s_{2}\left(\sigma_{2}\right)\right)}^{\varepsilon_{n+1}} \subset \widetilde{G}_{\varphi\left(\eta, s_{1}\left(\sigma_{1}\right), s_{2}\left(\sigma_{2}\right)\right)}^{\varepsilon_{n}} .
\end{aligned}
$$

Hence $\widetilde{w}_{n}\left(x, \varphi\left(\eta, s_{1}\left(\sigma_{1}\right), s_{2}\left(\sigma_{2}\right)\right), s_{2}\left(\sigma_{2}\right)\right)$ is well-defined and $I_{3}\left(x, \eta, \widetilde{G}_{\eta}(\tau)\right)$ is well-defined.

Therefore $\widetilde{w}_{n+1}(x, \eta, t)$ is well-defined and holomorphic on $\{(x, \eta, t) \in$ $\left.\mathbf{C}^{3} ;|x| \leq r, \eta \in E_{+}\left(\theta, \rho-\sum_{j=0}^{n+1} \varepsilon_{j}\right), t \in \widetilde{G}_{\eta}^{\varepsilon_{n+1}}\right\}$. Moreover on $\{(x, \eta, t) \in$ 
$\left.\mathbf{C}^{3} ;|x| \leq r, \eta \in E_{+}\left(\theta, \rho-\sum_{j=0}^{n+1} \varepsilon_{j}\right), t \in \widetilde{G}_{\eta}\right\}$ we have the following representations:

$$
\begin{aligned}
I_{1}\left(x, \eta, \widetilde{G}_{\eta}(R)\right)= & \frac{\mathrm{c}}{\mathrm{b}}\left(\frac{\mathrm{a}}{\mathrm{b}}+x\right)^{-1} \int_{0}^{R}\left\{1+\mathrm{d}\left(|\eta|-R_{1}\right) e^{i \arg (\eta)}\right\}^{\mathrm{b} / \mathrm{d}} \widetilde{w}_{n}\left(x, \eta, \widetilde{G}_{\eta}\left(R_{1}\right)\right) \\
& \times \frac{(1+\mathrm{d} \eta) e^{i \arg (\eta)}}{\left\{1+\mathrm{d}\left(|\eta|-R_{1}\right) e^{i \arg (\eta)}\right\}^{2}} d R_{1}, \\
I_{2}\left(x, \eta, \widetilde{G}_{\eta}(R)\right)= & \frac{\mathrm{c}}{\mathrm{b}}\left(\frac{\mathrm{a}}{\mathrm{b}}+x\right)^{-1}\left\{1+\mathrm{d}(|\eta|-R) e^{i \arg (\eta)}\right\}^{\mathrm{b} / \mathrm{d}} \\
& \times \int_{0}^{R} \widetilde{w}_{n}\left(x,\left(|\eta|-R+R_{1}\right) e^{i \arg (\eta)}, \widetilde{G}_{\left.\left(|\eta|-R+R_{1}\right) e^{i \arg (\eta)}\left(R_{1}\right)\right)}\right. \\
& \times \frac{e^{i \arg (\eta)}}{1+\mathrm{d}(|\eta|-R) e^{i \arg (\eta)} d R_{1},} \\
I_{3}\left(x, \eta, \widetilde{G}_{\eta}(R)\right)= & \mathrm{c}\left(\frac{\mathrm{a}}{\mathrm{b}}+x\right)^{-1} \int_{0}^{R} \int_{0}^{R_{1}}\left\{1+\mathrm{d}\left(|\eta|-R_{1}\right) e^{i \arg (\eta)}\right\}^{\mathrm{b} / \mathrm{d}} \\
& \times \widetilde{w}_{n}\left(x,\left(|\eta|-R_{1}+R_{2}\right) e^{i \arg (\eta)}, \widetilde{G}_{\left.\left(|\eta|-R_{1}+R_{2}\right) e^{i \arg (\eta)}\left(R_{2}\right)\right)}\right. \\
& \times \frac{\left\{e^{i \arg (\eta)}\right\}^{2}}{\left\{1+\mathrm{d}\left(|\eta|-R_{1}\right) e^{i \arg (\eta)}\right\}^{2}} d R_{2} d R_{1}
\end{aligned}
$$

Let us estimate each $I_{i}\left(x, \eta, \widetilde{G}_{\eta}(R)\right)$.

On $I_{1}\left(x, \eta, \widetilde{G}_{\eta}(R)\right)$ : It follows from the assumption of the induction that

$$
\begin{aligned}
\left|\widetilde{w}_{n}\left(x, \eta, \widetilde{G}_{\eta}\left(R_{1}\right)\right)\right| \leq & C_{1} e^{\delta_{1}|\eta|}\left(M L K^{3}\right)^{n} \frac{1}{\left(1+|\eta|-R_{1}\right)^{n(1-p)}} \\
& \times \sum_{k=0}^{n}\left(\begin{array}{l}
n \\
k
\end{array}\right)\left\{\frac{1}{\delta_{1}}\left(1+\frac{1}{1-p}\right)\right\}^{n-k} \frac{(1+|\eta|)^{k}}{k !(1-p)^{k}}
\end{aligned}
$$

which implies that

$$
\begin{aligned}
&\left|I_{1}\left(x, \eta, \widetilde{G}_{\eta}(R)\right)\right| \\
& \leq C_{1} e^{\delta_{1}|\eta|}\left(M L K^{3}\right)^{n+1} \sum_{k=0}^{n}\left(\begin{array}{l}
n \\
k
\end{array}\right)\left\{\frac{1}{\delta_{1}}\left(1+\frac{1}{1-p}\right)\right\}^{n-k} \frac{(1+|\eta|)^{k+1}}{k !(1-p)^{k}} \\
& \times \int_{0}^{R} \frac{1}{\left(1+|\eta|-R_{1}\right)^{n(1-p)+2-p}} d R_{1} \\
& \leq C_{1} e^{\delta_{1}|\eta|}\left(M L K^{3}\right)^{n+1} \sum_{k=0}^{n}\left(\begin{array}{l}
n \\
k
\end{array}\right)\left\{\frac{1}{\delta_{1}}\left(1+\frac{1}{1-p}\right)\right\}^{n-k} \frac{(1+|\eta|)^{k+1}}{k !(1-p)^{k}}
\end{aligned}
$$




$$
\begin{aligned}
& \times \frac{1}{(n+1)(1-p)} \frac{1}{(1+|\eta|-R)^{(n+1)(1-p)}} \\
\leq & C_{1} e^{\delta_{1}|\eta|}\left(M L K^{3}\right)^{n+1} \frac{1}{(1+|\eta|-R)^{(n+1)(1-p)}} \\
& \times \sum_{k=0}^{n}\left(\begin{array}{l}
n \\
k
\end{array}\right)\left\{\frac{1}{\delta_{1}}\left(1+\frac{1}{1-p}\right)\right\}^{n-k} \frac{(1+|\eta|)^{k+1}}{(k+1) !(1-p)^{k+1}} \\
= & C_{1} e^{\delta_{1}|\eta|}\left(M L K^{3}\right)^{n+1} \frac{1}{(1+|\eta|-R)^{(n+1)(1-p)}} \\
& \times \sum_{k=1}^{n+1}\left(\begin{array}{c}
n \\
k-1
\end{array}\right)\left\{\frac{1}{\delta_{1}}\left(1+\frac{1}{1-p}\right)\right\}^{n+1-k} \frac{(1+|\eta|)^{k}}{k !(1-p)^{k}}
\end{aligned}
$$

On $I_{2}\left(x, \eta, \widetilde{G}_{\eta}(R)\right)$ : The assumption of the induction implies that

$$
\begin{aligned}
\mid \widetilde{w}_{n}(x, & \left(|\eta|-R+R_{1}\right) e^{i \arg (\eta)}, \widetilde{G}_{\left.\left(|\eta|-R+R_{1}\right) e^{i \arg (\eta)}\left(R_{1}\right)\right) \mid} \\
\leq & C_{1} e^{\delta_{1}|\eta|} e^{-\delta_{1} R} e^{\delta_{1} R_{1}}\left(M L K^{3}\right)^{n} \frac{1}{\left(1+|\eta|-R+R_{1}-R_{1}\right)^{n(1-p)}} \\
& \times \sum_{k=0}^{n}\left(\begin{array}{l}
n \\
k
\end{array}\right)\left\{\frac{1}{\delta_{1}}\left(1+\frac{1}{1-p}\right)\right\}^{n-k} \frac{\left(1+|\eta|-R+R_{1}\right)^{k}}{k !(1-p)^{k}} \\
= & C_{1} e^{\delta_{1}|\eta|} e^{-\delta_{1} R} e^{\delta_{1} R_{1}}\left(M L K^{3}\right)^{n} \frac{1}{(1+|\eta|-R)^{n(1-p)}} \\
& \times \sum_{k=0}^{n}\left(\begin{array}{l}
n \\
k
\end{array}\right)\left\{\frac{1}{\delta_{1}}\left(1+\frac{1}{1-p}\right)\right\}^{n-k} \frac{\left(1+|\eta|-R+R_{1}\right)^{k}}{k !(1-p)^{k}} .
\end{aligned}
$$

Hence we obtain that

$$
\begin{aligned}
& \left|I_{2}\left(x, \eta, \widetilde{G}_{\eta}(R)\right)\right| \\
& \leq C_{1} e^{\delta_{1}|\eta|}\left(M L K^{3}\right)^{n}(M L K) \frac{1}{(1+|\eta|-R)^{(n+1)(1-p)}} \\
& \quad \times \sum_{k=0}^{n}\left(\begin{array}{l}
n \\
k
\end{array}\right)\left\{\frac{1}{\delta_{1}}\left(1+\frac{1}{1-p}\right)\right\}^{n-k} e^{-\delta_{1} R} \int_{0}^{R} e^{\delta_{1} R_{1}} \frac{\left(1+|\eta|-R+R_{1}\right)^{k}}{k !(1-p)^{k}} d R_{1} \\
& \leq C_{1} e^{\delta_{1}|\eta|}\left(M L K^{3}\right)^{n+1} \frac{1}{(1+|\eta|-R)^{(n+1)(1-p)}} \\
& \quad \times \sum_{k=0}^{n}\left(\begin{array}{l}
n \\
k
\end{array}\right)\left\{\frac{1}{\delta_{1}}\left(1+\frac{1}{1-p}\right)\right\}^{n-k} \frac{1}{\delta_{1}} \frac{(1+|\eta|)^{k}}{k !(1-p)^{k}}
\end{aligned}
$$

(see $(4.11))$. 
On $I_{3}\left(x, \eta, \widetilde{G}_{\eta}(R)\right)$ : By the assumption of the induction, we have

$$
\begin{aligned}
& \mid \widetilde{w}_{n}\left(x,\left(|\eta|-R_{1}+R_{2}\right) e^{i \arg (\eta)}, \widetilde{G}_{\left.\left(|\eta|-R_{1}+R_{2}\right) e^{i \arg (\eta)}\left(R_{1}\right)\right) \mid}\right. \\
& \leq C_{1} e^{\delta_{1}|\eta|} e^{-\delta_{1} R_{1}} e^{\delta_{1} R_{2}}\left(M L K^{3}\right)^{n} \frac{1}{\left(1+|\eta|-R_{1}+R_{2}-R_{2}\right)^{n(1-p)}} \\
& \quad \times \sum_{k=0}^{n}\left(\begin{array}{l}
n \\
k
\end{array}\right)\left\{\frac{1}{\delta_{1}}\left(1+\frac{1}{1-p}\right)\right\}^{n-k} \frac{\left(1+|\eta|-R_{1}+R_{2}\right)^{k}}{k !(1-p)^{k}} \\
& =C_{1} e^{\delta_{1}|\eta|} e^{-\delta_{1} R_{1}} e^{\delta_{1} R_{2}}\left(M L K^{3}\right)^{n} \frac{1}{\left(1+|\eta|-R_{1}\right)^{n(1-p)}} \\
& \quad \times \sum_{k=0}^{n}\left(\begin{array}{l}
n \\
k
\end{array}\right)\left\{\frac{1}{\delta_{1}}\left(1+\frac{1}{1-p}\right)\right\}^{n-k} \frac{\left(1+|\eta|-R_{1}+R_{2}\right)^{k}}{k !(1-p)^{k}},
\end{aligned}
$$

which implies that

$$
\begin{aligned}
\mid I_{3}(x, & \left.\eta, \widetilde{G}_{\eta}(R)\right) \mid \\
\leq & C_{1} e^{\delta_{1}|\eta|}\left(M L K^{3}\right)^{n}\left(M L K^{2}\right) \sum_{k=0}^{n}\left(\begin{array}{l}
n \\
k
\end{array}\right)\left\{\frac{1}{\delta_{1}}\left(1+\frac{1}{1-p}\right)\right\}^{n-k} \\
& \times \int_{0}^{R} \frac{1}{\left(1+|\eta|-R_{1}\right)^{n(1-p)+2-p}} e^{-\delta_{1} R_{1}} \int_{0}^{R_{1}} e^{\delta_{1} R_{2}} \\
\leq & C_{1} e^{\delta_{1}|\eta|}\left(M L K^{3}\right)^{n+1} \sum_{k=0}^{n}\left(\begin{array}{l}
n \\
k
\end{array}\right)\left\{\frac{1}{\delta_{1}}\left(1+\frac{1}{1-p}\right)\right\}^{n-k} \frac{1}{\delta_{1}} \frac{(1+|\eta|)^{k}}{k !(1-p)^{k}} \\
& \times \int_{0}^{R} \frac{1}{\left(1+|\eta|-R_{1}\right)^{k}} d R_{2} d R_{1} \\
\leq & C_{1} e^{\delta_{1}|\eta|}\left(M L K^{3}\right)^{n+1} \sum_{k=0}^{n}\left(\begin{array}{l}
n \\
k
\end{array}\right)\left\{\frac{1}{\delta_{1}}\left(1+\frac{1}{1-p}\right)\right\}^{n-k} \frac{1}{\delta_{1}} \frac{(1+|\eta|)^{k}}{k !(1-p)^{k}} \\
& \times \frac{1 R_{1}}{(n+1)(1-p)} \frac{(4.11))}{(1+|\eta|-R)^{(n+1)(1-p)}} \\
\leq & C_{1} e^{\delta_{1}|\eta|}\left(M L K^{3}\right)^{n+1} \frac{1}{(1+|\eta|-R)^{(n+1)(1-p)}} \\
& \times \sum_{k=0}^{n}\left(\begin{array}{l}
n \\
k
\end{array}\right)\left\{\frac{1}{\delta_{1}}\left(1+\frac{1}{1-p}\right)\right\}^{n-k} \frac{1}{\delta_{1}} \frac{1}{1-p} \frac{(1+|\eta|)^{k}}{k !(1-p)^{k}} .
\end{aligned}
$$


Therefore we have

$$
\begin{aligned}
& \left|\widetilde{w}_{n+1}\left(x, \eta, \widetilde{G}_{\eta}(R)\right)\right| \\
& \leq C_{1} e^{\delta_{1}|\eta|}\left(M L K^{3}\right)^{n+1} \frac{1}{(1+|\eta|-R)^{(n+1)(1-p)}} \\
& \times\left[\sum_{k=1}^{n+1}\left(\begin{array}{c}
n \\
k-1
\end{array}\right)\left\{\frac{1}{\delta_{1}}\left(1+\frac{1}{1-p}\right)\right\}^{n+1-k} \frac{(1+|\eta|)^{k}}{k !(1-p)^{k}}\right. \\
& \left.+\sum_{k=0}^{n}\left(\begin{array}{l}
n \\
k
\end{array}\right)\left\{\frac{1}{\delta_{1}}\left(1+\frac{1}{1-p}\right)\right\}^{n-k}\left(\frac{1}{\delta_{1}}+\frac{1}{\delta_{1}} \frac{1}{1-p}\right) \frac{(1+|\eta|)^{k}}{k !(1-p)^{k}}\right] \\
& =C_{1} e^{\delta_{1}|\eta|}\left(M L K^{3}\right)^{n+1} \frac{1}{(1+|\eta|-R)^{(n+1)(1-p)}} \\
& \times\left[\sum_{k=1}^{n+1}\left(\begin{array}{c}
n \\
k-1
\end{array}\right)\left\{\frac{1}{\delta_{1}}\left(1+\frac{1}{1-p}\right)\right\}^{n+1-k} \frac{(1+|\eta|)^{k}}{k !(1-p)^{k}}\right. \\
& \left.+\sum_{k=0}^{n}\left(\begin{array}{l}
n \\
k
\end{array}\right)\left\{\frac{1}{\delta_{1}}\left(1+\frac{1}{1-p}\right)\right\}^{n+1-k} \frac{(1+|\eta|)^{k}}{k !(1-p)^{k}}\right] \\
& =C_{1} e^{\delta_{1}|\eta|}\left(M L K^{3}\right)^{n+1} \frac{1}{(1+|\eta|-R)^{(n+1)(1-p)}} \\
& \times\left[\left\{\frac{1}{\delta_{1}}\left(1+\frac{1}{1-p}\right)\right\}^{n+1}+\frac{(1+|\eta|)^{n+1}}{(n+1) !(1-p)^{n+1}}\right. \\
& \left.+\sum_{k=1}^{n}\left\{\left(\begin{array}{c}
n \\
k-1
\end{array}\right)+\left(\begin{array}{l}
n \\
k
\end{array}\right)\right\}\left\{\frac{1}{\delta_{1}}\left(1+\frac{1}{1-p}\right)\right\}^{n+1-k} \frac{(1+|\eta|)^{k}}{k !(1-p)^{k}}\right] \\
& =C_{1} e^{\delta_{1}|\eta|}\left(M L K^{3}\right)^{n+1} \frac{1}{(1+|\eta|-R)^{(n+1)(1-p)}} \\
& \times \sum_{k=0}^{n+1}\left(\begin{array}{c}
n+1 \\
k
\end{array}\right)\left\{\frac{1}{\delta_{1}}\left(1+\frac{1}{1-p}\right)\right\}^{n+1-k} \frac{(1+|\eta|)^{k}}{k !(1-p)^{k}},
\end{aligned}
$$

which implies the lemma for $n+1$. The proof is completed.

\section{$\S 7 . \quad$ Some Remarks}

In Cases (3) and (4), we assumed the additional conditions (1.12) and (1.13), respectively. In this section, we consider the case where these conditions are removed. 
In Case (3). We remove the condition (1.12). Therefore $\Re\left(-\mathrm{b} e^{i \theta}\right)<0$ and $\mathrm{c} \neq 0$. The formal Borel transform $v(x, \eta)$ of the formal solution satisfies the integral equation (5.5). For the sake of simplicity, we assume $f(x, y)=f(x)$ (therefore $h(x, \eta) \equiv 0$ ), and consider the equation obtained by removing the fourth and fifth terms in the right hand side of (5.5):

$$
\begin{aligned}
v(x, \eta)= & f\left(\left(\frac{\mathrm{a}}{\mathrm{b}}+x\right) e^{-\mathrm{b} \eta}-\frac{\mathrm{a}}{\mathrm{b}}\right) \\
& +\frac{\mathrm{c}}{\mathrm{a}+\mathrm{b} x} \int_{0}^{\eta} e^{\mathrm{b}(\eta-t)} v\left(\left(\frac{\mathrm{a}}{\mathrm{b}}+x\right) e^{-\mathrm{b}(\eta-t)}-\frac{\mathrm{a}}{\mathrm{b}}, t\right) d t .
\end{aligned}
$$

This integral equation is equivalent to the initial value problem of the following first order linear partial differential equation:

$$
\begin{gathered}
\left\{D_{\eta}+(\mathrm{a}+\mathrm{b} x) D_{x}\right\} v(x, \eta)=\frac{\mathrm{c}}{\mathrm{a}+\mathrm{b} x} v(x, \eta), \\
v(x, 0)=f(x) .
\end{gathered}
$$

Hence we can solve this equation explicitly as

$$
v(x, \eta)=f\left(\left(\frac{\mathrm{a}}{\mathrm{b}}+x\right) e^{-\mathrm{b} \eta}-\frac{\mathrm{a}}{\mathrm{b}}\right) \times \exp \left[\frac{\mathrm{c}}{\mathrm{b}(\mathrm{a}+\mathrm{b} x)}\left(e^{\mathrm{b} \eta}-1\right)\right] .
$$

Therefore if $\Re\left(-\mathrm{b} e^{i \theta}\right)<0$ and $\mathrm{c} \neq 0$, this $v(x, \eta)$ does not have the exponential growth estimate. It has a growth estimate of the form $\exp \left[\delta e^{p|\eta|}\right]$ with $p=$ $\Re\left(\mathrm{b} e^{i \theta}\right)>0$.

By the above argument, it seems that the solution $v(x, \eta)$ of the original equation (5.5) may not satisfy the condition (BS) and that the formal solution $u(x, y)$ may not be Borel summable unless (1.12) is satisfied.

In Case (4). We remove the condition (1.13). Therefore $\Re(-\mathrm{b} / \mathrm{d}) \leq-1$ and $\mathrm{c} \neq 0$. The formal Borel transform $v(x, \eta)$ of the formal solution satisfies the integral equation (6.4). We assume $f(x, y)=f(x)$ and consider the equation which is obtained in a similar manner to Case (3):

$$
\begin{aligned}
v(x, \eta)= & f\left(\left(\frac{\mathrm{a}}{\mathrm{b}}+x\right)(1+\mathrm{d} \eta)^{-\mathrm{b} / \mathrm{d}}-\frac{\mathrm{a}}{\mathrm{b}}\right) \\
+ & \frac{\mathrm{c}}{\mathrm{b}}\left(\frac{\mathrm{a}}{\mathrm{b}}+x\right)^{-1}(1+\mathrm{d} \eta)^{\mathrm{b} / \mathrm{d}} \\
& \quad \times \int_{0}^{\eta}(1+\mathrm{d} t)^{-\mathrm{b} / \mathrm{d}} v\left(\left(\frac{\mathrm{a}}{\mathrm{b}}+x\right)\left(\frac{1+\mathrm{d} \eta}{1+\mathrm{d} t}\right)^{-\mathrm{b} / \mathrm{d}}-\frac{\mathrm{a}}{\mathrm{b}}, t\right) d t .
\end{aligned}
$$

This equation is equivalent to the following initial value problem: 


$$
\begin{gathered}
\left\{(1+\mathrm{d} \eta) D_{\eta}+(\mathrm{a}+\mathrm{b} x) D_{x}\right\} v(x, \eta)=\frac{\mathrm{c}(1+\mathrm{d} \eta)}{\mathrm{a}+\mathrm{b} x} v(x, \eta), \\
v(x, 0)=f(x) .
\end{gathered}
$$

First we assume $\mathrm{b} \neq \mathrm{d}$ and solve this equation. We can obtain the explicit form of the solution as follows:

$$
\begin{aligned}
v(x, \eta)= & f\left(\left(\frac{\mathrm{a}}{\mathrm{b}}+x\right)(1+\mathrm{d} \eta)^{-\mathrm{b} / \mathrm{d}}-\frac{\mathrm{a}}{\mathrm{b}}\right) \\
& \times \exp \left[\frac{\mathrm{c}}{\mathrm{b}-\mathrm{d}} \frac{1}{\mathrm{a}+\mathrm{b} x}\left\{(1+\mathrm{d} \eta)^{\mathrm{b} / \mathrm{d}}-(1+\mathrm{d} \eta)\right\}\right] .
\end{aligned}
$$

Therefore if $\Re(-\mathrm{b} / \mathrm{d})<-1$, this $v(x, \eta)$ has a growth order of the form $\exp \left(\delta|\eta|^{p}\right)$ with $p=\Re(\mathrm{b} / \mathrm{d})>1$ and does not satisfy the condition (BS).

Next we consider the case $\Re(-\mathrm{b} / \mathrm{d})=-1$. Let us assume, for example, $\mathrm{b}=\mathrm{d}$. Then we can solve the equation (7.5) explicitly as

$$
v(x, \eta)=f\left(\left(\frac{\mathrm{a}}{\mathrm{b}}+x\right)(1+\mathrm{b} \eta)^{-1}-\frac{\mathrm{a}}{\mathrm{b}}\right) \times \exp \left[\frac{\mathrm{c}}{\mathrm{b}} \frac{1}{\mathrm{a}+\mathrm{b} x}(1+\mathrm{b} \eta) \log (1+\mathrm{b} \eta)\right] .
$$

Therefore, in this case also, this $v(x, \eta)$ does not have the exponential growth estimate.

By the above argument, if we remove the condition (1.13), it seems that the solution $v(x, \eta)$ of the original equation (6.4) may not satisfy the condition (BS) and that the formal solution $u(x, y)$ may not be Borel summable.

\section{References}

[1] Balser, W., From divergent power series to analytic functions, Lecture Notes in Math. 1582, Springer-Verlag, 1994.

[2] Balser, W., Formal power series and linear systems of meromorphic ordinary differential equations, Universitext, Springer-Verlag, 2000.

[3] Balser, W. and Miyake, M., Summability of formal solutions of certain partial differential equations, Acta Sci. Math. (Szeged), 65 (1999), 543-551.

[4] Gérard, R. and Tahara, H., Singular Nonlinear Partial Differential Equations, Vieweg, 1996.

[5] Hibino, M., Divergence Property of Formal Solutions for Singular First Order Linear Partial Differential Equations, Publ. RIMS, Kyoto Univ., 35 (1999), 893-919.

[6] Hibino, M., Gevrey Asymptotic Theory for Singular First Order Linear Partial Differential Equations of Nilpotent Type - Part I -, Preprint.

[7] Luts, D. A., Miyake, M. and Schäfke, R., On the Borel summability of divergent solutions of the heat equation, Nagoya Math. J., 154 (1999), 1-29.

[8] Miyake, M., Borel summability of divergent solutions of the Cauchy problem to nonKowalevskian equations, in Proc. Conference on PDE and their applications, (Wuhan, China 5-9 April 1999, edited by Chen Hua and L. Rodino), World Scientific, 1999, pp. 225-239. 
[9] Miyake, M., Newton polygons and formal Gevrey indices in the Cauchy-Goursat-Fuchs type equations, J. Math. Soc. Japan, 43 (1991), 303-330.

[10] Miyake, M. and Hashimoto, Y., Newton Polygons and Gevrey Indices for Linear Partial Differential Operators, Nagoya Math. J., 128 (1992), 15-47.

[11] Miyake, M. and Shirai, A., Convergence of Formal Solutions of First Order Singular Nonlinear Partial Differential Equations in Complex Domain, to appear in Ann. Polon. Math. (Krakov), special volume to the memory of B. Ziemian, 2000.

[12] Oshima, T., On the Theorem of Cauchy-Kowalevski for First Order Linear Differential Equation with Degenerate Principal Symbol, Proc. Japan Acad, 49 (1973), 83-87

[13] Ōuchi, S., Genuine solutions and formal solutions with Gevrey type estimates of nonlinear partial differential equations, J. Math. Sci. Univ. Tokyo, 2 (1995), 375-417.

[14] Shirai, A., Maillet Type Theorem for Nonlinear Partial Differential Equations and Newton Polygons, to appear in J. Math. Soc. Japan.

[15] Wasow, W., Asymptotic expansions for ordinary differential equations, Interscience Publ. John Wiley and Sons, Inc., New York, London, Sydney, 1965. 